\title{
2 Evidence for skeletal muscle fiber type-specific expressions 3 of mechanosensors
}

\author{
4 Sebastian Mathes ${ }^{1} \cdot$ Mathias Vanmunster $^{2} \cdot$ Wilhelm Bloch $^{1} \cdot$ Frank Suhr ${ }^{1,2}$ (I)
}

\section{Abstract}

Received: 19 September 2018 / Revised: 10 January 2019 / Accepted: 23 January 2019

c) Springer Nature Switzerland AG 2019

Mechanosensors govern muscle tissue integrity and constitute a subcellular structure known as costameres. Costameres physically link the muscle extracellular matrix to contractile and signaling 'hubs' inside muscle fibers mainly via integrins and are localized beneath sarcolemmas of muscle fibers. Costameres are the main mechanosensors converting mechanical cues into biological events. However, the fiber type-specific costamere architecture in muscles is unexplored. We hypothesized that fiber types differ in the expression of genes coding for costamere components. By coupling laser microdissection to a multiplex tandem qPCR approach, we demonstrate that type 1 and type 2 fibers indeed show substantial differences in their mechanosensor complexes. We confirmed these data by fiber type population-specific protein analysis and confocal microscopy-based localization studies. We further show that knockdown of the costamere gene integrin-linked kinase (Ilk) in muscle precursor cells results in significantly increased slow-myosin-coding $M y h 7$ gene, while the fast-myosin-coding genes $M y h 1, M y h 2$, and Myh4 are downregulated. In parallel, protein synthesis-enhancing signaling molecules (p-mTOR ${ }^{\text {Ser2448, }}$ $p<0.05$; p-P70S6K ${ }^{\text {Thr389 }}$, tendency with $p<0.1$ ) were reduced upon Ilk knockdown. However, overexpression of slow typeinducing NFATc1 in muscle precursor cells did not change $I l k$ or other costamere gene expressions. In addition, we demonstrate fiber type-specific costamere gene regulation upon mechanical loading and unloading conditions. Our data imply that costamere genes, such as $I l k$, are involved in the control of muscle fiber characteristics. Further, they identify costameres as muscle fiber type-specific loading management 'hubs' and may explain adaptation differences of muscle fiber types to mechanical (un)loading.

\section{Introduction}

Mammalian skeletal muscle (SkM) is composed of heterogeneous SkM fibers reflected by the high variability of morphological, biochemical, mechanical, and metabolic phenotypes of each individual SkM fiber. The following classification is widely accepted [1]: type 1 fibers are oxidative and slow twitch/fatigue resistant (aerobic metabolism);

Electronic supplementary material The online version of this article (https://doi.org/10.1007/s00018-019-03026-3) contains supplementary material, which is available to authorized users.

Frank Suhr

Frank.suhr@kuleuven.be

1 Department of Molecular and Cellular Sport Medicine, German Sport University Cologne, Cologne, Germany

2 Exercise Physiology Research Group, Department of Movement Sciences, Biomedical Sciences Group, KU Leuven, Tervuursevest 101, Bus 1500, 3001 Leuven, Belgium type 2A fibers are glycolytic and fast twitch/fatigue resistant (aerobic/anaerobic metabolism); type 2X/B fibers are glycolytic and fast twitch/fatigable (anaerobic metabolism). Importantly, all SkM fiber types diverge in terms of mechanical impacts they generate and tolerate [1] and adapt differentially to loading [2].

SkM fibers bear an intrinsic capacity to sense mechanical stimuli (mechanosensing) and convert these into biochemical events (mechanotransduction) that regulate the integrity and functionality of SkM [3]. Mechanical loading conditions, e.g., resistance training, result in SkM hypertrophy, while SkM disuse, e.g., immobilization, causes SkM atrophy [4]. Defined subcellular structures, known as costameres (Cstms), convert mechanical forces into biological signaling pathways triggering physiological adaptions of individual SkM fibers to mechanical loading [5]. However, it remains unknown if this Cstms-dependent mechanical impact conversion operates fiber type-specifically. Cstms are multiprotein complexes that localize beneath the sarcolemma and physically link to transmembrane heterodimeric integrin

\begin{tabular}{|l|l|l|l|l|}
\hline Journal : Large 18 & Article No : 3026 & Pages : 18 & MS Code : CMLS-D-18-01314 & Dispatch : 29-1-2019 \\
\hline
\end{tabular}


receptors thus connecting the SkM extracellular matrix (ECM) microenvironment with contractile and signaling hubs within individual SkM fibers [6]. Therefore, Cstms function as 'hubs' to convert mechanical impacts reaching individual SkM fibers into biological signals to mediate SkM fiber adaptations to these impacts [5]. The knowledge about fiber type-specific Cstms will enhance our comprehension of processes governing differential adaptations between type 1 and type 2 fibers.

The composition of Cstms in SkM fibers appears to be diverse [6] and their spatiotemporal assembly determines downstream adaptations in the respective SkM fibers [7, 8]. The integrin receptor complex (IRC) of Cstms defines a mechano-sensitive unit composed of integrins [mainly $\alpha 5$ (ITGA5), $\alpha 7$ (ITGA7), and $\beta 1 \mathrm{D}$ (ITGB1D) subunits] and the integrin-linked kinase (ILK)-pinch-parvin (IPP) complex [7]. ILK directly interacts with ITGB1D in SkM and is a mechanosensor critical for SkM integrity. Its knockdown in SkM tissue resulted in a progressive muscular dystrophy [8]. In addition, ITGA5 [9] and ITGA7 [10] deletions result in severe muscle diseases similar to muscle dystrophies in humans. ILK and its IPP partners are of special interest, because (1) they constitute a 'physical bridge' between the interior and exterior environments of the SkM and (2) they convert and amplify integrin-mediated signals since integrins lack enzymatic activity. Therefore, ILK and the IRC/IPP are as central players responsible for mechanotransduction to maintain and secure SkM tissue integrity and adaptation. The IRC/IPP complex contains additional components regulated by mechanical factors. Proteins, such as talin (TLN [11]), vinculin (VCL [12]) or kindlin-2 (FERMT2/KIND2 [13]), support Cstms functions to manage SkM functionality.

Individual type 1 and type 2 fibers show variable properties [1] and individual adaptation potential to loading [14]. Individual SkM fiber types diverge in amount and molecular composition of cytoskeleton scaffolds in the way that mechanical peak power values increase orderly from type 1 to type $2 \mathrm{~A}$ and 2X/B [15-17] fibers. In terms of loading, individual fiber types show variable extent of transcriptional regulation [18], phosphorylation pattern, and protein synthesis [2]. Therefore, we hypothesized (1) that type 1 and type 2 fibers differ in their Cstms expressions under sedentary conditions, (2) that altered mechanical loading changes Cstms gene expressions in type 1 and type 2 fibers differentially and (3) that $I l k$ is involved in the control of defined $M y h$ gene expressions in muscle precursor cells. We used the laser microdissection technique to collect pure type 1 and type 2 fiber populations and analyzed their Cstms-related gene expression profiles by a multiplex tandem qPCR approach. We found that type 1 and type 2 fibers show highly divergent Cstms gene expression patterns under basal conditions, which remodel under (un)loading conditions. We confirmed our gene expression results by fiber type-specific protein analysis and confocal microscopy-based localization studies. Furthermore, we knocked down $I l k$ in muscle precursor cells and found significantly increased slow-myosin-coding $M y h 7$ gene expressions accompanied by downregulations of fast-myosin-coding $M y h 1, M y h 2$, and Myh4. In contrast, overexpression of the slow twitch-inducing transcription factor nuclear factor of activated T cells (NFATc1) did not change Cstms gene expressions. Our data provide evidence that individual SkM fibers possess distinct molecular tools to manage mechanical (un)loading conditions and explain that type 1 and type 2 fibers adapt differentially to mechanical (un)loading due to fiber type-specific spatiotemporal assemblies of Cstms 'decision hubs' to govern downstream signals. Finally, we identify Ilk as a SkM fiber-determining Cstms component.

\section{Methods}

\section{Mice and interventions}

Twelve-week-old male C57BL/6J mice were maintained in a conventional animal facility at $22-24{ }^{\circ} \mathrm{C}$ of the German Sport University under a 12-h light/dark cycle in individual cages supplemented with water and chow ad libitum. Experimental procedures were approved by the State Office of North Rhine-Westphalia (84-02.04.2011.A097). Mice were randomly divided into three groups: (1) sedentary control (control, $n=8$ ), mice of this group did not receive any specific intervention; (2) whole-body vibration (WBV; $n=8)$, mice of this group were subjected to WBV [19, 20] for seven consecutive days. WBV is an established approach to induce SkM hypertrophy can precisely be standardized $\mathbf{A Q 3} ;$ $[21,22]$. WBV training was performed for $30 \mathrm{~min}^{-1} \mathrm{yy}^{-1}$ on a vibration platform (amplitude $=25 \mathrm{~Hz}$, incline $=5^{\circ}$ ). $\mathrm{SkM}$ mass-gaining effects were proven by significantly increased cross-sectional areas (CSA) of SkM fibers (figure S1A); (3) hind limb immobilization (IML; $n=8$ ), mice of this group were hind limb-immobilized for seven consecutive days by treating quadriceps and gastrocnemius muscle heads with a total of $2 \mathrm{U}$ of Dysport ${ }^{\circledR}$ (botulinum toxin type A; Ipsen, UK). Measurement of gait dysfunction was important to monitor the non-usage of hind limbs during the intervention period. Hence, we assessed gait dysfunction by multiobserver inventory and was determined on scores according to previous studies [23] ranging between " 0 " (completely disabled) to "10" (normal), for details, see figure S1B. SkM mass-losing effects were proven by a significant decrease in CSA of SkM fibers (figure S1A).

\section{Laser microdissection}

Laser microdissection (LMD) was performed using the PALM Laser-MicroBeam system (Zeiss). The system used 
a pulsed UV laser $(337 \mathrm{~nm})$, which was coupled via the epifluorescence port into a microscope and focused trough the objective. The microscope stage was under motorized control and a frame grabber enabled a real-time observation of the microscopic image on a computer screen. Laser manipulation of tissue was performed through a graphical user interface that allowed selection for subsequent microdissection of fibers in a collection device. For LMD, fibers of interest from mouse soleus muscle (containing a relatively high portion of oxidative type 1 fibers compared to other SkMs [24] enabling us to collect sufficient amounts of both fiber types for downstream analyses) were identified and selected through a $40 \times$ PlanNeofluar objective. UV laser was focused such that a 2- $\mu$ m-diameter gap surrounding the fiber was produced. Upon isolation of fibers, a high-energy pulse of the focused laser beam just below the focal plane of the tissue section catapulted the fiber into the cap of a 200$\mu \mathrm{L}$ reaction tube (Fig. 3a). For subsequent RNA analysis, individual type 1 or type 2 fibers (comprising $2 \mathrm{~A}, 2 \mathrm{X}$ and $2 \mathrm{~B}$ fibers) corresponding to a total surface area of $3 \times 10^{6} \mu \mathrm{m}^{2}$ were laser-microdissected and collected in $20 \mu \mathrm{L}$ GITC buffer to preserve RNA integrity. To reach the required amount of $3 \times 10^{6} \mu \mathrm{m}^{2}, 10$ - $\mu \mathrm{m}$-thick tissue sections were freshly prepared, mounted on membrane-coated PEN slides (Zeiss), ATPase-stained, dehydrated and LMD was continued. For subsequent protein analysis, fibers corresponding to a total surface area of $4 \times 10^{6} \mu \mathrm{m}^{2}$ were laser-microdissected and collected in $20 \mu \mathrm{L}$ RIPA buffer and protease inhibitor. Individual collection tubes were stored at $-80{ }^{\circ} \mathrm{C}$ and for further downstream analysis.

The integrity and yield of protein molecules were evaluated by 'scrape' tests to determine whether ATPase staining negatively influenced protein biochemistry. A total of five soleus cross sections were mounted on a glass slide, exposed for $10 \mathrm{~min}$ at RT and subsequently scraped from the slide. Another five sections were subjected to the same protocol, except that after RT exposure ATPase staining was performed. We compared unstained (USC) and stained scrape control (SSC) samples with $20 \mu \mathrm{g}$ of purified total protein isolated from frozen soleus tissue. We visually demonstrate that ATPase staining did not significantly affect the protein yield (Fig. 4a).

\section{Multiplex PCR}

Complete volumes of cDNA reverse transcribed from lasermicrodissected fibers were subjected to 17-plex PreAmp PCR. Multiplex PCR for type-I and type-II fibers was performed by mixing a final reaction volume of $50 \mu \mathrm{L}$ including the following components: (1) $25 \mu \mathrm{L}$ Platinum Multiplex PCR Master Mix (Applied Biosystems) including DNA polymerase and dNTPs; (2) $100 \mathrm{nM}$ primer mix consisting of 17 primer sets (forward and reverse, see supplement table 1) targeting the cDNA of 17 different genes of interests (GOI)s; (3) $10 \mu \mathrm{L}$ of cDNA; (4) $5 \mu \mathrm{L}$ nuclease-free $\mathrm{H}_{2} \mathrm{O}$ to reach a final reaction volume of $50 \mu \mathrm{L}$. The reaction was performed on a PCR cycler with the following protocol: initial denaturation $\left(2 \mathrm{~min}\right.$ at $\left.95{ }^{\circ} \mathrm{C}\right)$ followed by 15 PreAmp cycles including denaturation $\left(30 \mathrm{~s}\right.$ at $\left.95^{\circ} \mathrm{C}\right)$, annealing $(90 \mathrm{~s}$ at $59{ }^{\circ} \mathrm{C}$ ) and extension $\left(40 \mathrm{~s}\right.$ at $\left.72{ }^{\circ} \mathrm{C}\right)$. Final extension was performed for $10 \mathrm{~min}$ at $72{ }^{\circ} \mathrm{C}$. Afterwards cDNA was cooled to $4{ }^{\circ} \mathrm{C}$ and stored on ice. To decrease the concentration of primer and dNTPs as well as of inhibiting products from PreAmp reaction, cDNA PreAmp reaction volume was diluted 1:4.5 in $1 \times$ TE buffer and subsequently subjected to qPCR. cDNA input was standardized given that complete cDNA prepared from a defined laser-microdissected fiber area of $3 \times 10^{6} \mu \mathrm{m}^{2}$ was used. For uniformity assays, $50 \mathrm{ng}$, $25 \mathrm{ng}$, and $12.5 \mathrm{ng}$ cDNA were pre-amplified with 17-plex PCR and subsequently subjected to qPCR.

\section{Statistics}

All statistics were performed using Prism6 (GraphPad). Differences between type 1 and type 2 fibers were assessed using unpaired Student's $t$ test. Differences between groups were assessed using one-way ANOVA with Bonferroni corrected post hoc test. Levels of significance were $* p<0.05$, $* * p<0.01$, and $* * * p<0.001$.

\section{Supplementary methods}

Detailed information on tissue collection, immunofluorescence, ATPase staining, immunohistochemistry, RNA extraction, real-time RT-qPCR, RNA gel electrophoresis, cDNA synthesis, DNA gel electrophoresis, DNA extraction and sequencing, protein extraction, NuPAGE SDS-PAGE, western blot, Coomassie staining and buffers can be found in the supplements.

\section{Results}

\section{Components of the Cstms network show fiber type-dependent localizations}

To test our overall hypothesis that the components of Cstms show SkM fiber type-dependent patterns, we first used confocal microscopy to localize individual Cstms proteins in type 1 vs. type 2 SkM fibers with specific antibodies. In the soleus muscle of sedentary control mice, we studied the presence of IPP components ILK, PARVB, PINCH-1, of IRC components ITGA7 and ITGB1D as well as accessory molecules TLN, VCL and beta-XIN, a protein known to have important functions in cardiac muscle integrity and that stabilizes the sarcomeric architecture via binding and

\begin{tabular}{|l|l|l|l|l|}
\hline Journal : Large 18 & Article No : 3026 & Pages : 18 & MS Code : CMLS-D-18-01314 & Dispatch : 29-1-2019 \\
\hline
\end{tabular}


crosslinking F-actin $[25,26]$. These localization studies confirmed our hypothesis that Cstms components show a SkM fiber type-dependent localization (Fig. 1). Specifically, ILK, VCL, and ITGA7 localize predominantly in glycolytic type 2 fibers. On the contrary, PINCH-1, TLN, and beta-XIN localize predominantly in oxidative type 1 fibers. PARVB and ITGB1D do not show specific distributions. These data demonstrate that oxidative and glycolytic SkM fiber types differ in their respective assemblies of Cstms components.

\section{Cstms components accumulate heterogeneously between type 1 and type 2 fibers}

To further explore the protein levels observed by confocal localization studies, we stained soleus muscles from sedentary control mice with a specific Cstms antibodies and developed the respective signal by DAB, while we used immunofluorescence in parallel to stain type $1 \mathrm{SkM}$ fibers (MyHC1) on the same section. As demonstrated in Fig. 2a, this staining technique is suitable to stain different SkM fiber type components. Type 1 fibers are represented by a clear fluorescent signal, while Ilk (as an example) is represented by the DAB signal. Using this approach, we determined the relative protein densities in type 1 vs. type 2 SkM fibers. Figure $2 \mathrm{~b}$ shows significantly increased protein levels of ILK, VCL, ITGA7, ITGB1D and PARVB in glycolytic type 2 fibers. On the contrary, PINCH-1, TLN and beta-XIN are expressed at significantly higher levels in oxidative type 1 fibers. These data confirm our confocal microscopy-based localization studies with clear further evidence. Components of Cstms show SkM fiber type-dependent expression patterns.

\section{Type 1 and type 2 fibers differ in Cstms protein amounts}

In addition to the qualitative and semi-quantitative analyses of Cstms components in situ by means of confocal microscopy and DAB-based immunohistochemistry, we studied Cstms component protein levels in a SkM fiber type-dependent manner by western blot. To this end, we first used a metachromatic ATPase staining technique modified and optimized for mouse soleus muscle [27]. Figure $3 \mathrm{~b}$ shows an overview of a metachromatically stained $10-\mu \mathrm{m}$ section of the mouse soleus muscle. A higher power view of this section in Fig. 3c shows oxidative type 1 fibers in dark blue, whereas glycolytic type $2 \mathrm{~A}$ show almost no staining and glycolytic type $2 \mathrm{~B}$ fiber reveal a light blue staining. This metachromatic ATPase staining allowed us to simultaneously distinguish between type 1 and type 2 fiber type populations with staining completed within $20 \mathrm{~min}$, thereby minimizing potential protein degradation of the native SkM tissue. The LMD procedure to collect
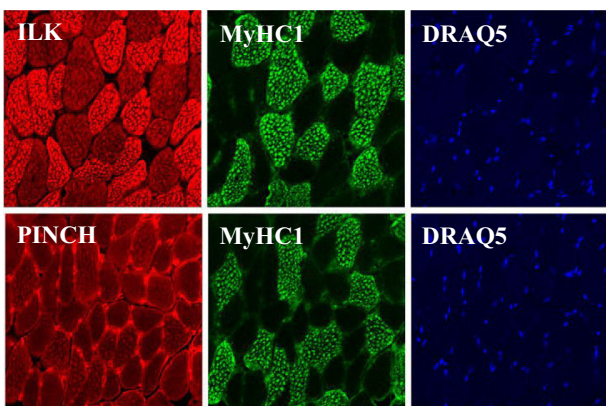

DRAQ5
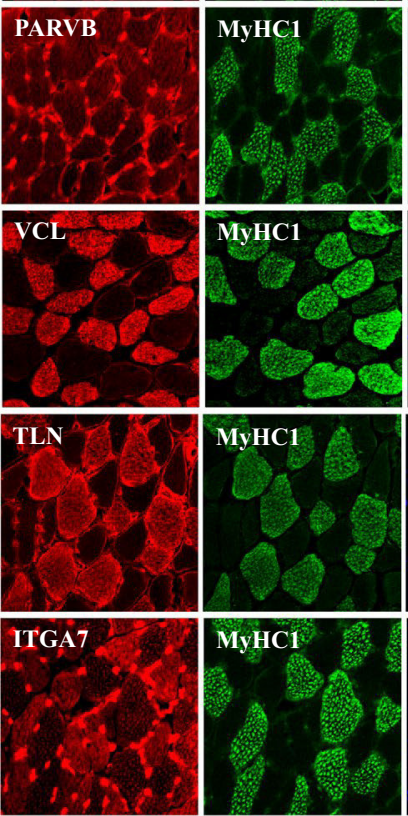

ITGB1
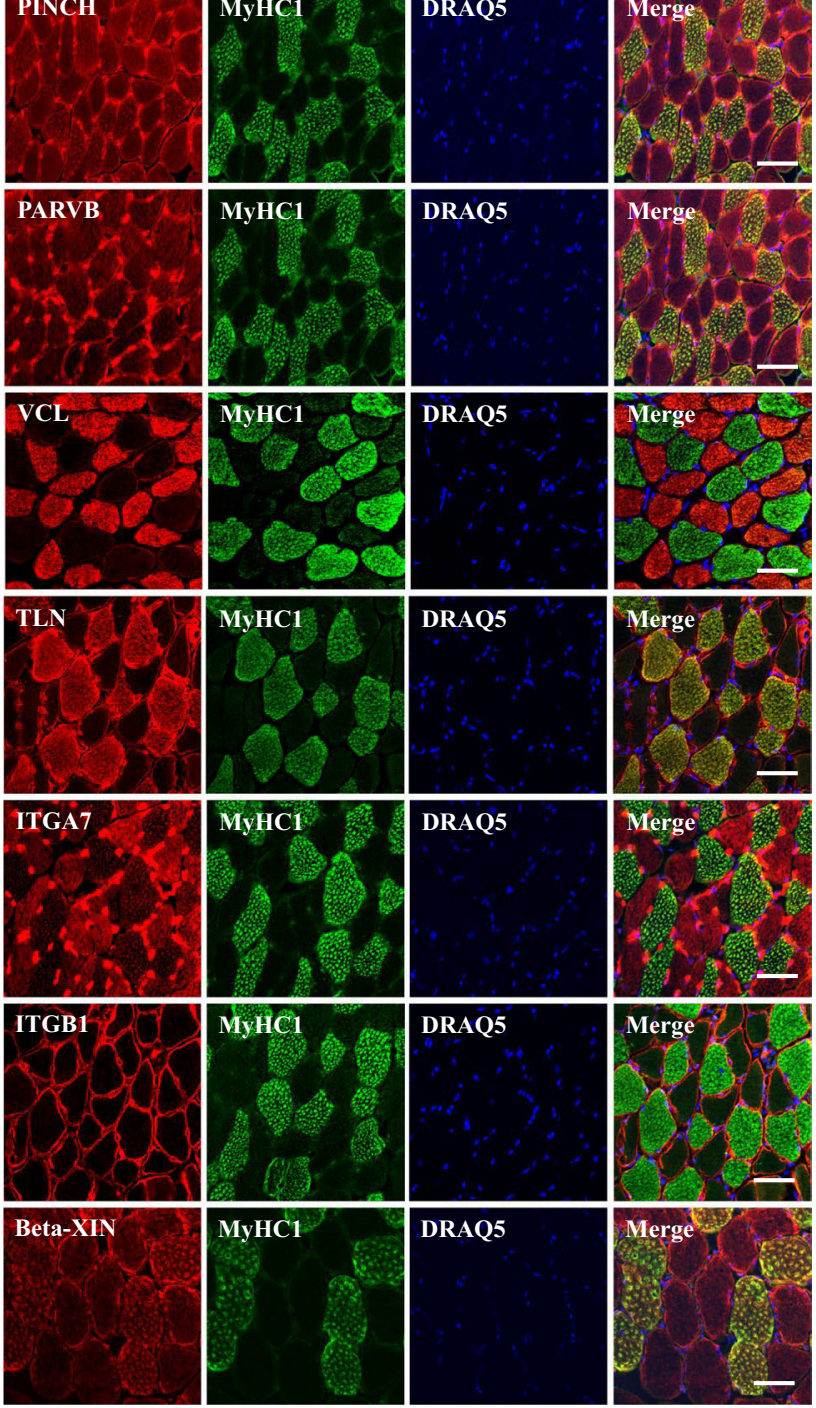

Fig. 1 Confocal microscopy-based Cstms component localizations. Representative images showing costameric proteins localized in skeletal muscle fibers. IF was performed on $10-\mu \mathrm{m}$ cross sections of mouse control soleus muscles with specific antibodies against ILK, PINCH-1, PARVB, VCL, TLN, ITGA7, ITGB1D, and beta-XIN (all shown in red). Type 1 fibers (shown in green) were stained with an antibody against myosin heavy chain 1 . Type 2 fibers (2A, 2B, 2X) are unstained (black). Nuclei were counterstained with DRAQ5 (shown in blue). PINCH-1, TLN and beta-XIN show type 1 fiber localizations, whereas ILK, VCL, and ITGA7 show predominantly type 2 fiber localizations. PARVB and ITGB1D do not show clear distributions. Scale bar $=50 \mu \mathrm{m}$

fiber type-specific populations is depicted in Fig. 3d-g. Using this approach, we were able to obtain purified type 1- and type 2-specific SkM fiber populations and to 

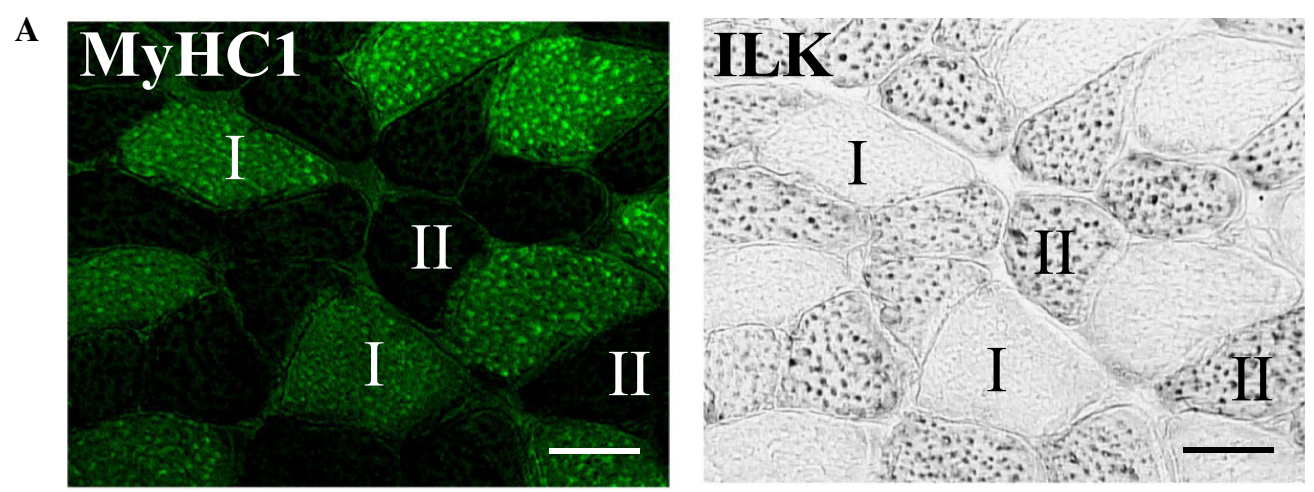

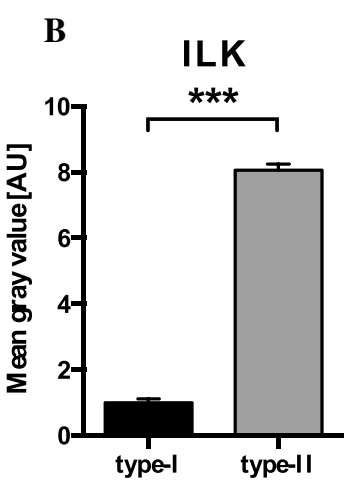

TLN

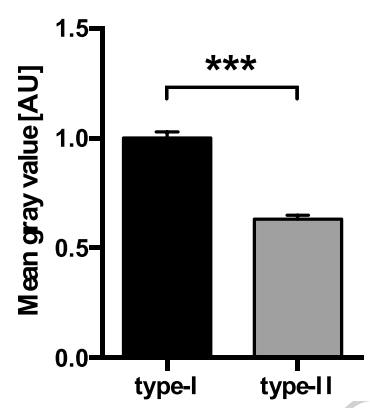

PINCH

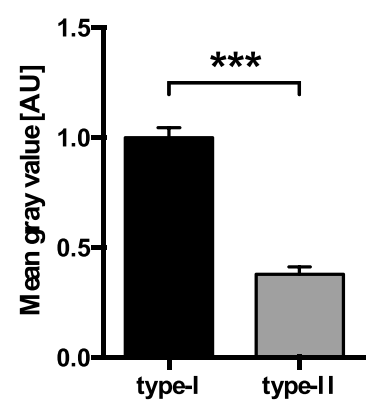

ITGA7

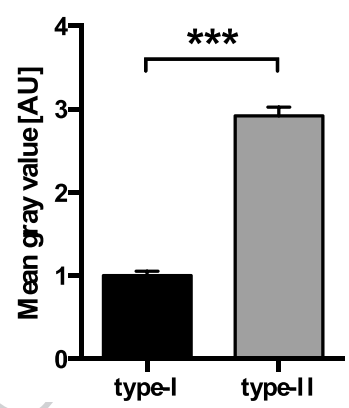

Fig. 2 Histology-based evidence of fiber type-dependent Cstms protein accumulations. a Fluorescence microscopy of $10-\mu \mathrm{m}$ cross sections of control soleus muscle showed MyHC 1 staining. Fibers were digitally labeled and after switch to light microscopy, DAB staining intensity (as shown representatively for ILK) was analyzed in type 1 and type 2 fibers. Scale bar $=20 \mu \mathrm{m}$. b Quantitative bar graphs of

extract the SkM proteins. To quantitatively visualize fiber type-specific protein levels, we developed a western blot method that enabled highly sensitive protein detection from approximately 1500 individual type 1 or type 2 fibers corresponding with a surface area of $4 \times 10^{6} \mu \mathrm{m}^{2}$. Because LMD of 1500 fibers yielded only low amounts of total protein ranging between $500 \mathrm{pg}$ and $1000 \mathrm{pg}$ (determined by dilution series, data not shown), we omitted protein determination and instead subjected total tissue homogenates

\section{PARVB}

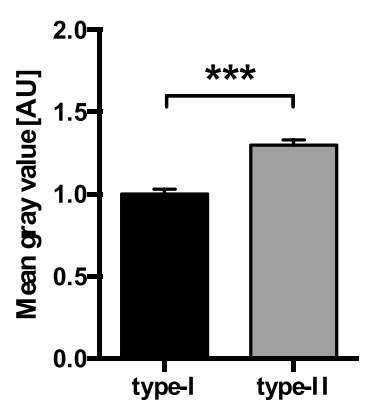

ITGB1D

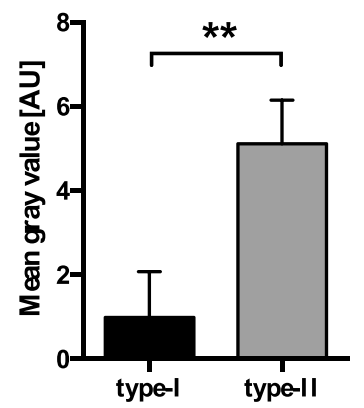

VCL

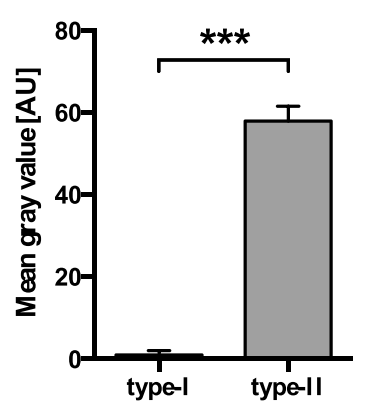

Beta-XIN

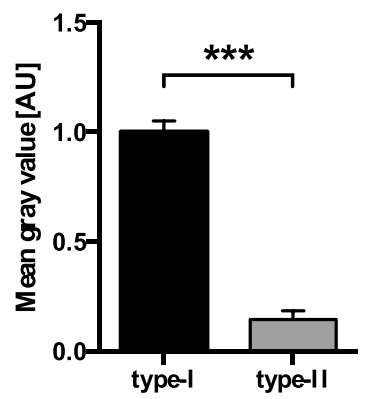

costameric protein expression, obtained from control mouse soleus muscles. Mean gray values are given in arbitrary units (AU) and were obtained from staining intensity of DAB-stained cross sections. Values are normalized to 'type 1'. Each bar represents mean \pm SEM of 150 myofibers of $n=3$ control mice. $* * * p<0.001$ between fiber types

from a standardized area of $4 \times 10^{6} \mu \mathrm{m}^{2}$ directly to gel electrophoresis. We detected $\alpha$-actinin- 2 at similar levels in both type 1 and type 2 fibers (Fig. 4b), which matched with previous reports [28]. Therefore, we used $\alpha$-actinin-2 as internal loading control. In general, most of the proteins examined showed robust differences in protein levels considering that short exposure times resulted in prominent band intensities in one fiber type, whereas the protein was almost undetectable in the other fiber type (demonstrated

\begin{tabular}{|l|l|l|l|l|}
\hline Journal : Large 18 & Article No : 3026 & Pages : 18 & MS Code : CMLS-D-18-01314 & Dispatch : 29-1-2019 \\
\hline
\end{tabular}




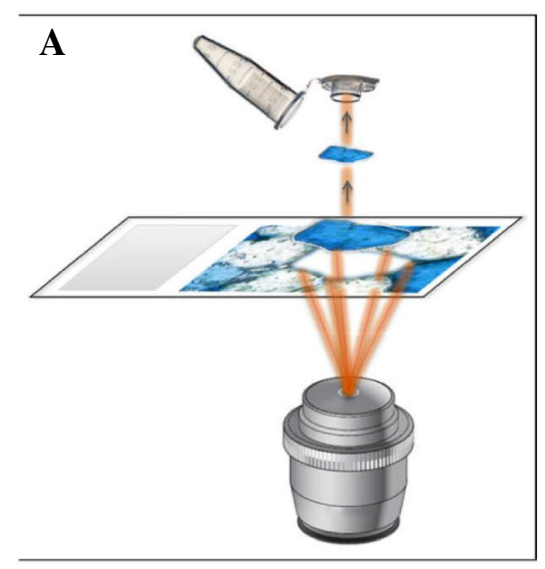

Principle of the LMD technique: A selected region of interest is catapulted by a high energy laser beam into the buffer solution-containing lid of a reaction tube.
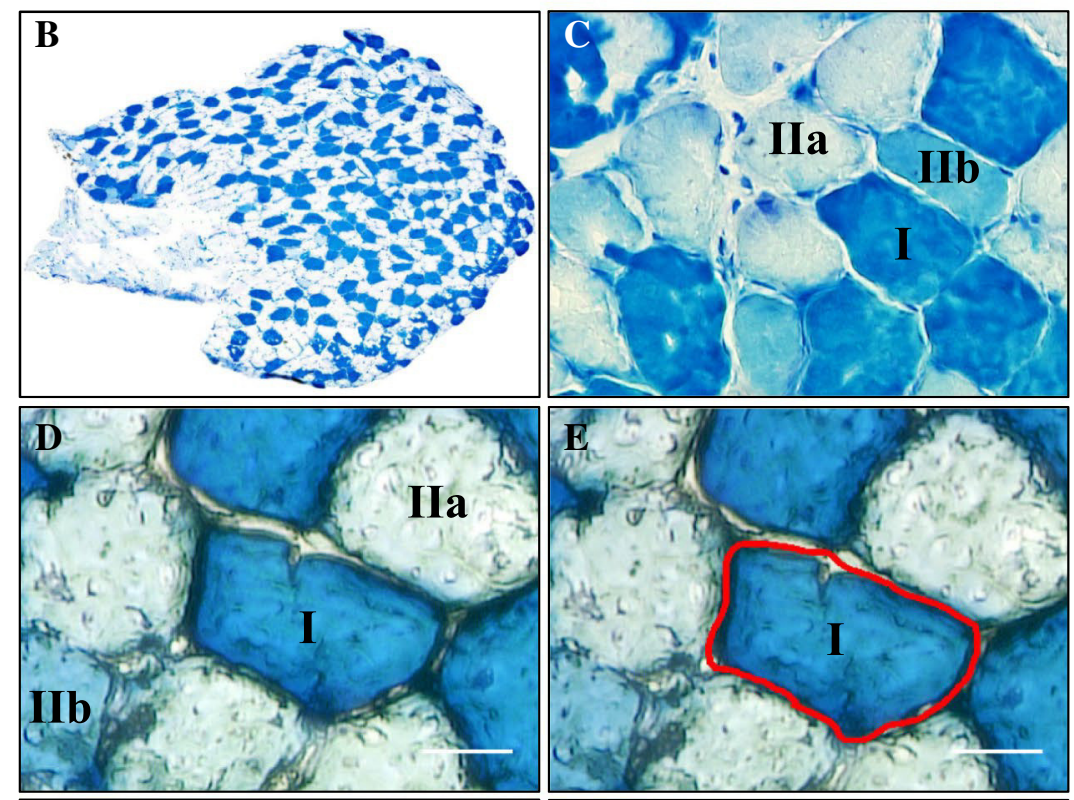

Type 1- and type 2 fiber type-specific populations from soleus muscles of control, WBV- and IMLtreated conditions were laser-microdissected upon metachromatic ATPase staining.
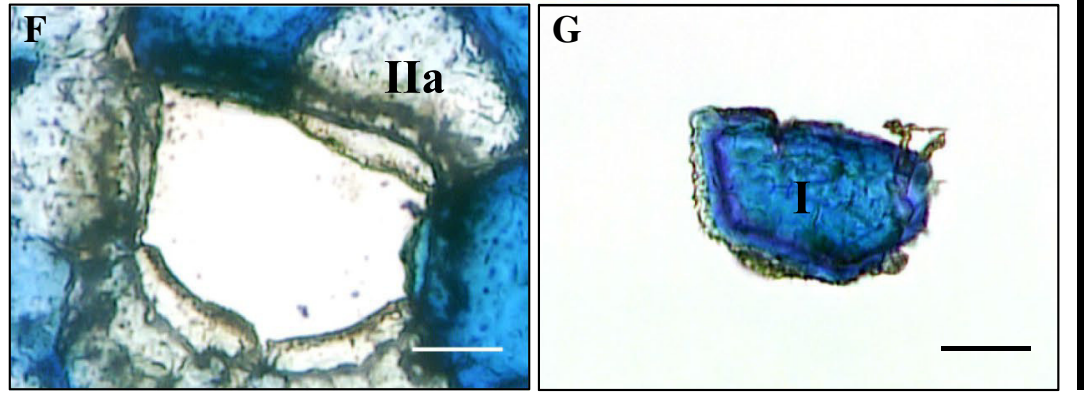

- Cstms protein analysis in type 1- and type 2-specific fiber populations: ILK, TLN, $\alpha$-actinin-2, $\alpha$-actinin-3, Actin, GAPDH

- Cstms gene analysis in type 1- and type 2-specific fiber populations: Myh1, Myh2, Myh4, Myh7, Ilk, Lims1, Parva, Fermt2, Xirp2, Tln1, Vcl, Itga7, Itgb1D, Fbxo32, Trim63, Rpl27, Rpl41

Fig. 3 Laser microdissection procedure of type 1 and type 2 fibers from mouse soleus muscles. a Principle of the collection procedure of SkM fiber type populations by the LMD technique. b Mouse soleus cross sections were stained with metachromatic ATPase staining technique to label type 1 and different type 2 fibers. c A magnification of a representative part of $\mathbf{b}$ showing the staining for different fiber types
(1, 2A, 2B). d Another zoomed part of a stained section, in which a type 1 fiber is digitally selected in e. Upon application of laser microdissection, the selected type 1 fiber was cut (f) and catapulted into a reagent tube (g). The workflow and the targets selected for protein and gene analysis, respectively, are also shown

\begin{tabular}{|l|l|l|l|l|}
\hline Journal : Large 18 & Article No : 3026 & Pages : 18 & MS Code : CMLS-D-18-01314 & Dispatch : 29-1-2019 \\
\hline
\end{tabular}



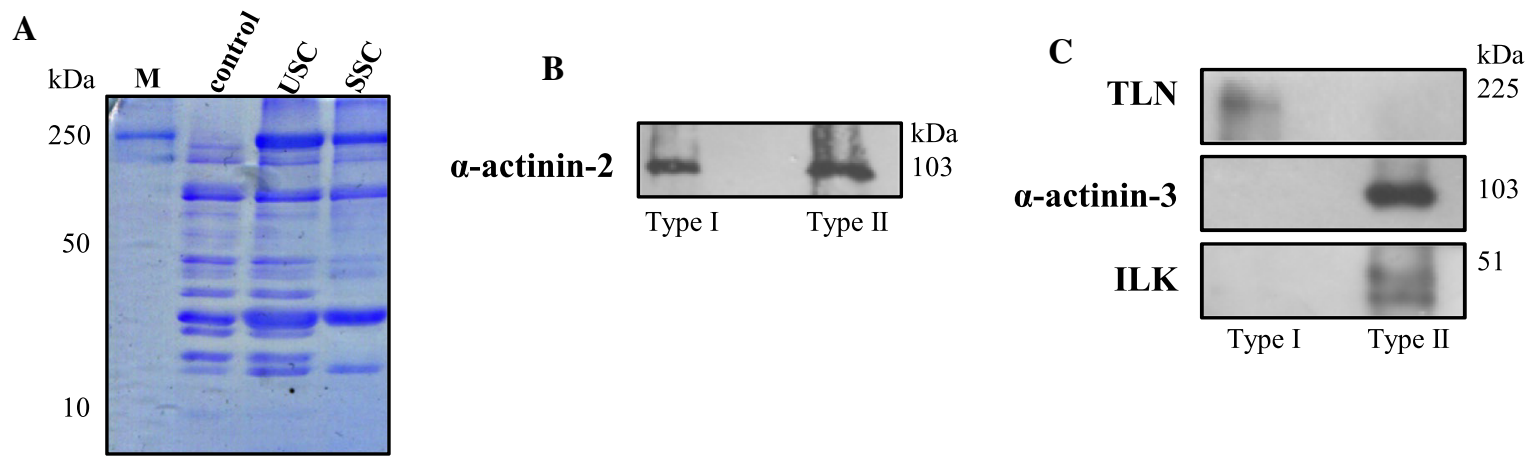

D
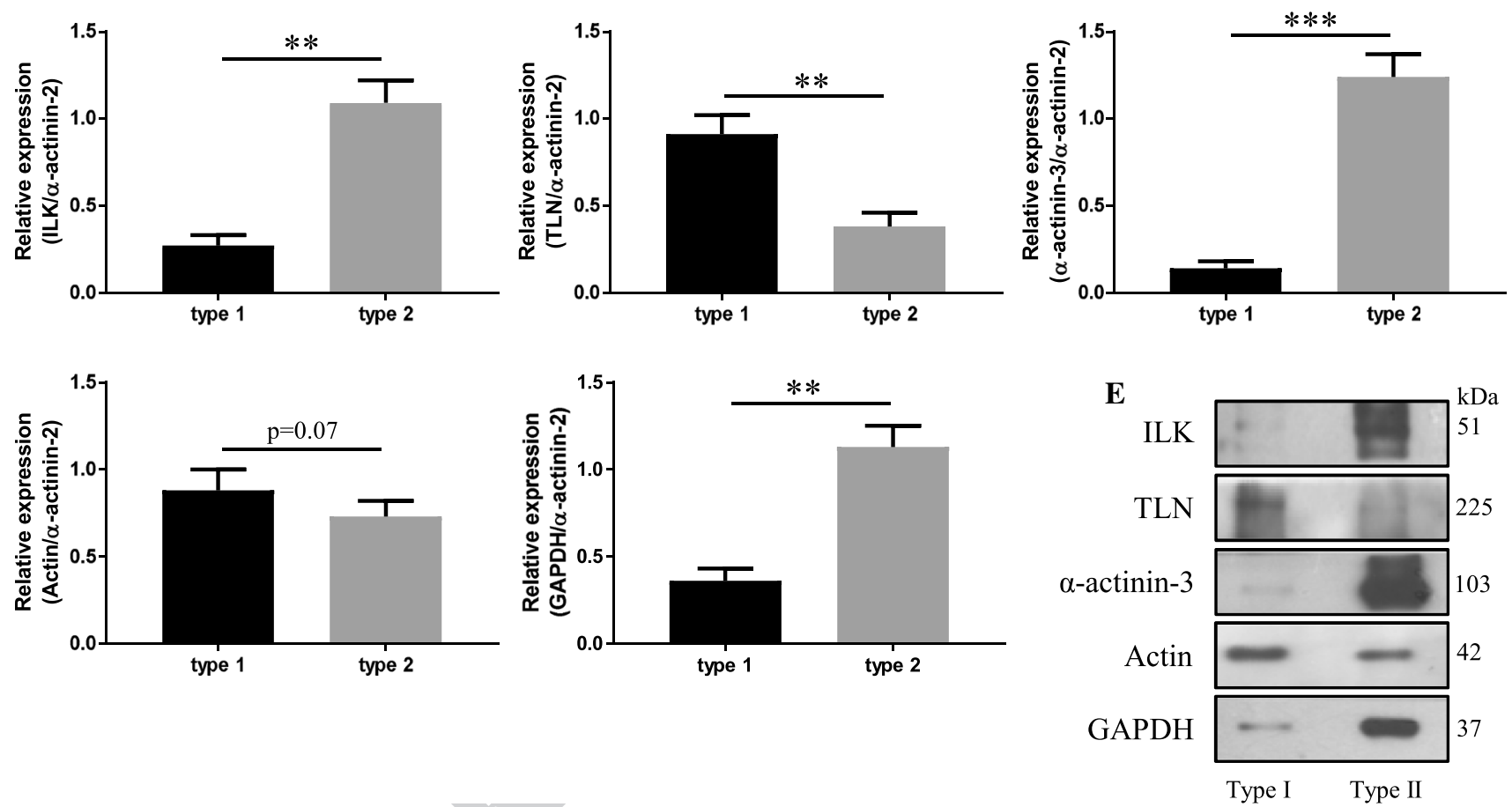

Fig. 4 Fiber type-specific protein analysis from laser-microdissected control soleus muscle. a Coomassie stain of $20 \mu \mathrm{g}$ of protein purified from fresh-frozen soleus (control), and from five unstained (USC) and stained (SSC) scrape controls shows no visual effect of ATPase staining on protein yield, b We used $\alpha$-actinin- 2 as internal loading control since this protein was evenly expressed in type 1 and type 2 fibers. c Decreased exposure time failed to detect proteins in type 2 (TLN) or type 1 ( $\alpha$-actinin-3, ILK) fibers. d Quantitative results for ILK, TLN, $\alpha$-actinin-3, actin, and GAPDH upon

for TLN, $\alpha$-actinin-3 and ILK, Fig. 4c). Figure $4 d$ shows quantitative analysis of ILK, TLN, $\alpha$-actinin- 3 , Actin, and GAPDH from soleus muscles of three sedentary control mice. ILK and $\alpha$-actinin- 3 were significantly enriched in type 2 fiber populations, whereas TLN was significantly enriched in type 1 fibers. Interestingly, the common housekeeping proteins actin and GAPDH showed fiber
NuPAGE SDS-PAGE of laser-microdissected type 1 and type 2, each corresponding to an area of $4 \times 10^{6} \mu \mathrm{m}^{2}$. Ilk and $\alpha$-actinin-3 were clearly expressed in type 2 fibers, whereas TLN was predominantly detected in type 1 fibers. Actin showed a tendency for higher values in type 1, while GAPDH was clearly higher in type 2 fibers. Due to this reason, we did neither use actin nor GAPDH as internal loading controls. e Representative western blots of ILK, TLN, $\alpha$-actinin-3, actin, and GAPDH are shown in type 1 and type 2 fibers. $* * p<0.01$, $* * * p<0.001 . M$ protein marker, $k D a$ kilo Dalton

type-specific enrichments. Actin levels tended to be higher in type 1 fibers, whereas GAPDH was significantly higher in type 2 fibers. Representative western blots are shown in Fig. 4e. These data provide evidence that defined Cstms components show SkM fiber type-specific differences in their quantities, which is consistent with our localization (Fig. 1) and histological (Fig. 2a, b) analyses.

\begin{tabular}{|l|l|l|l|l|}
\hline Journal : Large 18 & Article No : 3026 & Pages : 18 & MS Code : CMLS-D-18-01314 & Dispatch : 29-1-2019 \\
\hline
\end{tabular}



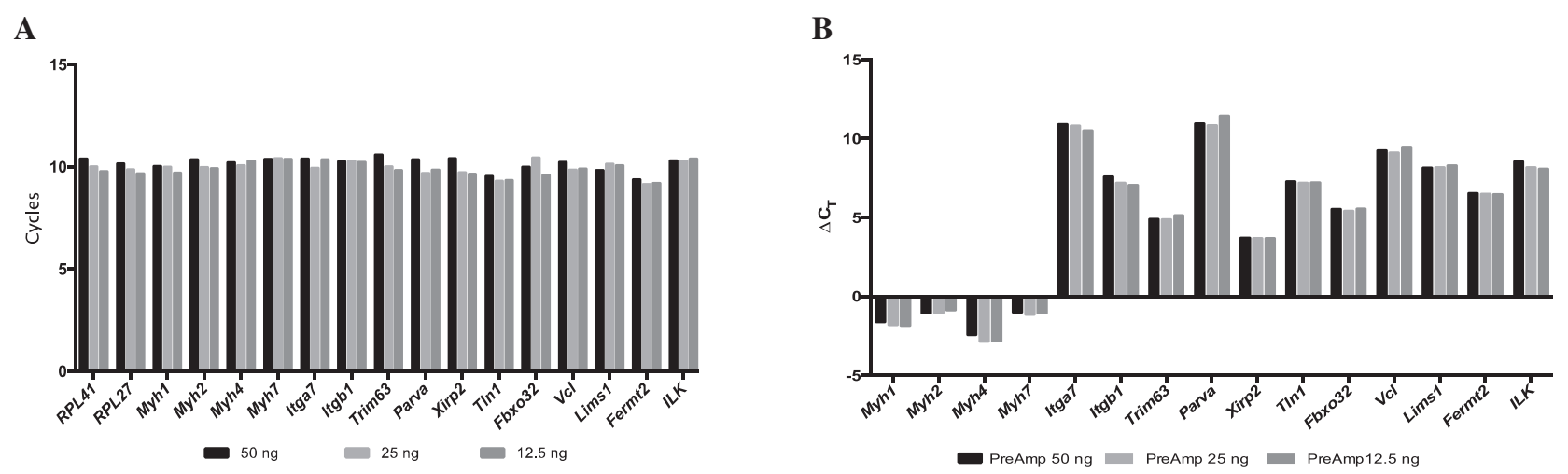

C

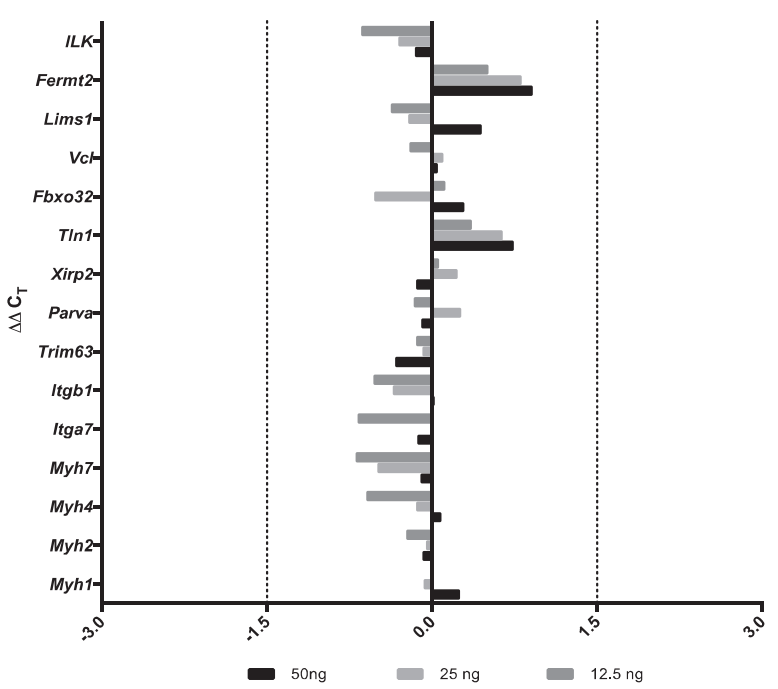

D
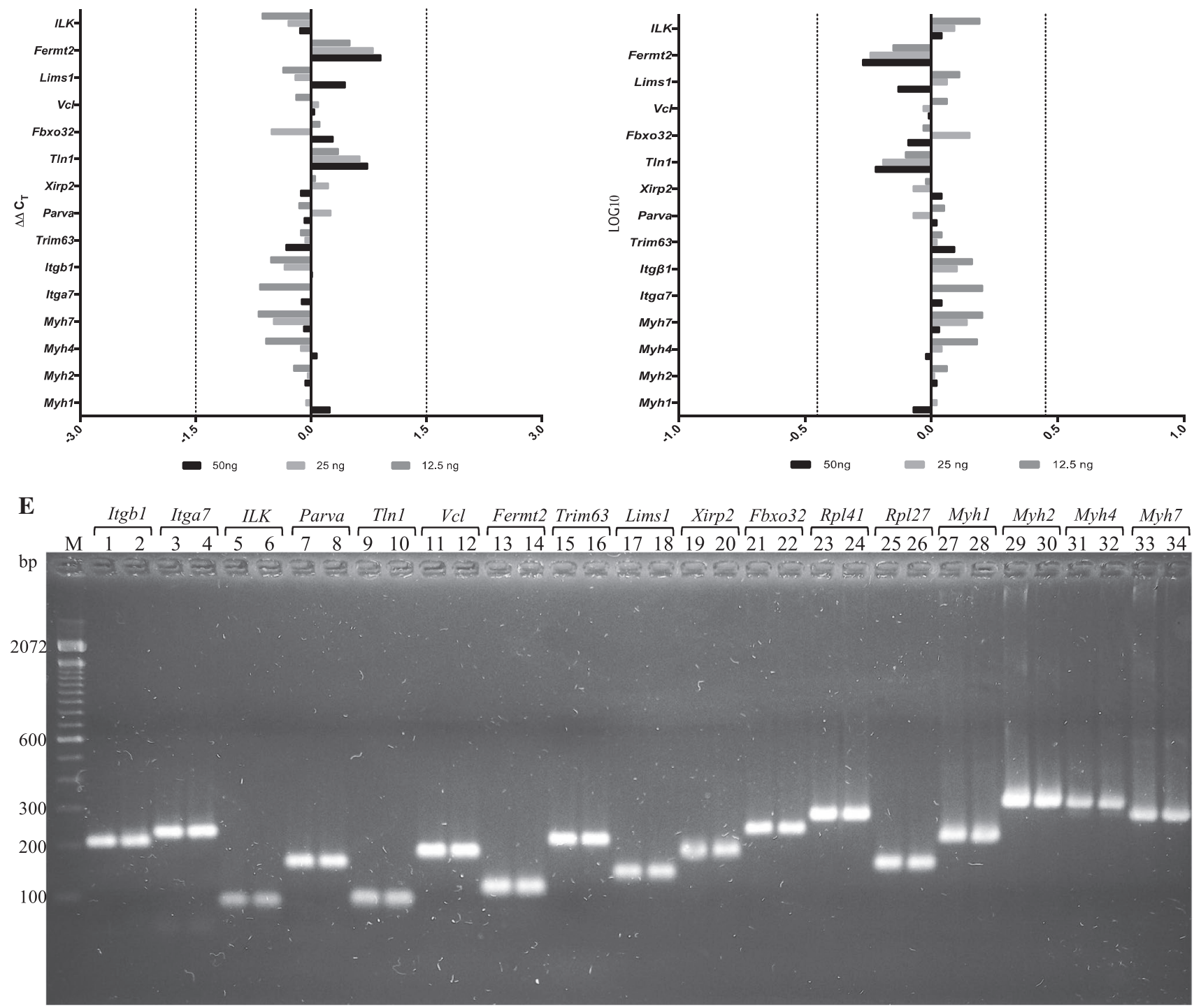

\section{Validation of the LMD approach and development of the multiplex tandem qPCR assay}

To further characterize differences in the levels of Cstms components between oxidative and glycolytic SkM fibers, we developed a highly sensitive multiplex tandem PCR assay. The low amount of RNA isolated from laser-microdissected fibers failed to reliably quantify gene expression via

\begin{tabular}{|l|l|l|l|l|}
\hline Journal : Large 18 & Article No : 3026 & Pages : 18 & MS Code : CMLS-D-18-01314 & Dispatch : 29-1-2019 \\
\hline
\end{tabular}


4Fig. 5 Test of uniformity of multiplex tandem PCR approach. a Interassay (PreAmp vs. no PreAmp) improvement of $C_{\mathrm{T}}$ achieved by three different 17-plex PreAmp reactions prepared from cDNA aliquots derived from control soleus muscle. Singleplex qPCR without cDNA PreAmp was performed using the same cDNA input (50 ng, $25 \mathrm{ng}$, or $12.5 \mathrm{ng}$ ) as for 17-plex PreAmp with subsequent singleplex qPCR. b Intra-assay variance of MT-PCR from three different PreAmp reactions (50 ng, $25 \mathrm{ng}, 12.5 \mathrm{ng}$ ) shows an overall coefficient of variation $(\mathrm{CV})$ of $2.7 \pm 2.7 \%$. c $\Delta C_{\mathrm{T}}$ values resulting from three different PreAmp reactions (50 ng, $25 \mathrm{ng}, 12.5 \mathrm{ng}$ ) were used to calculate $\Delta \Delta C_{\mathrm{T}}$ (PreAmp-multiplex vs. no PreAmp singleplex). No PreAmp singleplex was performed with the same cDNA inputs $(50 \mathrm{ng}, 25 \mathrm{ng}$, $12.5 \mathrm{ng}$ ) as for PreAmp-multiplex qPCR. $\Delta \Delta C_{\mathrm{T}}$ of \pm 0.9 shows uniformity of MT-PCR. d Uniformity of MT-PCR and no bias of relative expression are shown by $\log _{10}$ of $\Delta \Delta C_{\mathrm{T}}$ ranging in between \pm 0.27 . e DNA gel electrophoresis of qPCR product shows the absence of primer dimer formation. Agarose gel showed specificity of amplification in both singleplex qPCR (odd lane numbers) and MT-PCR (even lane numbers). GOIs were as follows: Itgb1 201-bp; Itga7 222bp; Ilk 85-bp; Parva 152-bp; Tln1 89-bp; Vcl 172-bp; Fermt2 107-bp; Trim63 201-bp; Lims1 130-bp; Xirp2 170-bp; Fbxo32 218-bp; Rpl41 260-bp; Rpl27 144-bp; Myh1 203-bp; Myh2 299-bp; Myh4 295-bp; $M y h 7$ 267-bp. $M$ DNA marker, $b p$ base pairs

standard RT-qPCR (data not shown). Since fiber typing prior to LMD was essential, we studied the three different staining protocols Oil Red O (figure S2), periodic acid-Schiff (PAS, figure S3) and ATPase (Fig. 3), the latter being the most promising. To quantify Cstms gene expressions upon ATPase staining-assisted LMD, we developed an assay in which we pre-amplified cDNA instead of RNA, which offered the advantage that RNA with RIN values $\approx 8$ could be used. This is appropriate and does not influence relative expressions obtained by RT-qPCR [29], why our approach is highly suitable to preserve RNA integrity for downstream gene expression analyses (figure S4).

To simultaneously quantify gene expression of 17 targets in a single laser-microdissected sample consisting of approximately 1200 fibers corresponding to a surface area of $3 \times 10^{6} \mu \mathrm{m}^{2}$, we reverse transcribed RNA and cDNA was pre-amplified using a 17-plex (multiplex) PCR. Preamplified (PreAmp) cDNA was then divided and subjected to (nested) real-time qPCR, referred to as multiplex tandem PCR (MT-PCR). MT-PCR contains the potential to amplify cDNA non-linearly due to primer dimer formation, underrepresentation of low-copy transcripts, and/or over-representation of high-copy transcripts. To validate our assay, we quantified gene expression from whole soleus muscle homogenates both with MT-PCR and singleplex RT-qPCR. MT-PCR revealed mean \pm SEM $C_{\mathrm{T}}$ improvement of $10.05 \pm 0.4$ cycles compared to singleplex RT-qPCR (Fig. 5a). The intra-assay variance of gene expression of 15 targets relative to calibrator genes Rpl41 and Rpl27 revealed a high reproducibility of the MT-PCR assay between varying amounts of cDNA (Fig. 5b). $\Delta C_{\mathrm{T}}$ variance of the 15 GOIs revealed an overall mean \pm SEM coefficient of variance $(\mathrm{CV})$ of $2.7 \pm 2.4 \%$, which perfectly matched previously reported
$\mathrm{CV}$ values of MT-PCRs [30]. $\Delta \Delta C_{\mathrm{T}}$ (multiplex vs. singleplex) values close to zero indicate ideal PreAmp uniformity and according to Noutsias et al. [30]. PreAmp uniformity can be assumed if $\Delta \Delta C_{\mathrm{T}}$ values range between \pm 1.5 or if $\log _{10}$ of $\Delta \Delta C_{\mathrm{T}}$ is between $\pm 0.452 . \Delta \Delta C_{\mathrm{T}}$ values obtained from our MT-PCR results taking the mean of Rpl41 and $R p l 27$ as internal calibrator and singleplex qPCR as controls revealed $\Delta \Delta C_{\mathrm{T}}$ values of \pm 0.9 (Fig. $5 \mathrm{c}$ ). $\log _{10}$ of $\Delta \Delta C_{\mathrm{T}}$ ranged between \pm 0.27 (Fig. 5d) confirming uniformity of PreAmp. Melt curve analysis (figure S4B and S4C) and DNA gel electrophoresis of qPCR amplicons revealed specificity of the amplification without primer dimer formations during MT-PCR (Fig. 5e).

\section{Cstms genes are differentially expressed in type 1 and type 2 fibers under sedentary conditions}

We laser-microdissected approximately 1200 type 1 and type 2 fibers, each corresponding to a surface area of $3 \times 10^{6} \mu \mathrm{m}^{2}$ from sedentary control mice.

Gene expression of four myosin heavy-chain isoforms (Myh1,Myh2, Myh4 and Myh7) demonstrated successful LMD of type 1 and type 2 fiber populations. Myhl (coding for MyHC 2X) expression was significantly higher in type 2 than in type 1 fiber (Fig. 6a). We found similar results for Myh2 (coding for MyHC 2A) expression (Fig. 6b) and Myh4 (coding for MyHC 2B) expressions (Fig. 6c). In contrast, $M y h 7$ (coding for MyHC 1) expression levels were significantly higher (Fig. 6d) in type 1 than in type 2 fibers.

The Cstms gene Ilk was significantly increased in type 2 compared to type 1 fibers (Fig. 6e). The ILK-related gene Lims1 (coding for Pinch-1) showed a tendency to increase in type 1 fibers (Fig. 6f), whereas Parva (coding for alphaparvin) expression was significantly more increased in type 1 compared to type 2 fibers (Fig. 6g). The integrin-activator Fermt2 (coding for kindlin-2) showed significantly higher levels in type 2 compared to type 1 fibers (Fig. 6h), a comparable pattern to Ilk. The actin-regulating Xirp2 gene (coding for beta-xin) showed significantly increased levels in type 1 fibers (Fig. 6i). The costameric Tln1 (coding for talin-1) and $\mathrm{Vcl}$ (coding for vinculin) genes showed significantly higher gene expressions in type 2 compared to type 1 fibers (Fig. 6j, $\mathrm{k}$, respectively). The two main integrin subunits Itga7 (coding for integrin $\alpha 7$ ) and Itgb1D (coding for integrin beta1D) showed significantly higher expression levels in type 2 compared to type 1 fibers (Fig. 61, m, respectively). The protein breakdown-associated genes Fbxo32 (coding for muscle atrophy F-box protein atrogin-1) did not show differential expression between fiber types (Fig. 6n), whereas Trim63 (coding for E3 ubiquitin-protein ligase Trim63) showed significantly higher levels in type 2 compared to type 1 fibers (Fig. 6o). The specificity of all MT-PCR amplifications from type 1 and type 2 fibers was confirmed by melt curve

\begin{tabular}{|l|l|l|l|l|}
\hline Journal : Large 18 & Article No : 3026 & Pages : 18 & MS Code : CMLS-D-18-01314 & Dispatch : 29-1-2019 \\
\hline
\end{tabular}




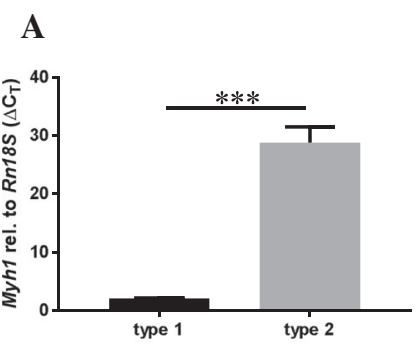

B

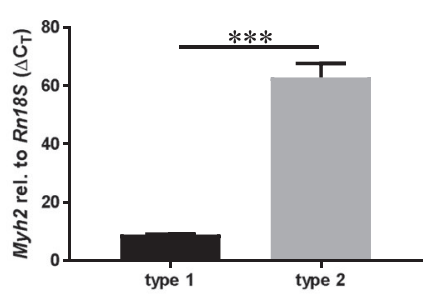

$\mathbf{E}$
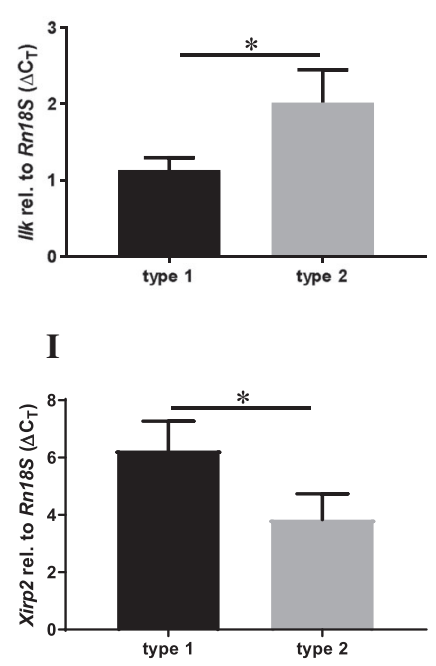

L

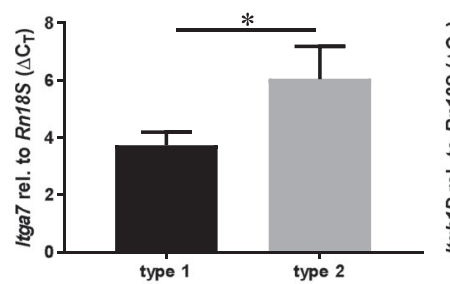

$\mathbf{N}$

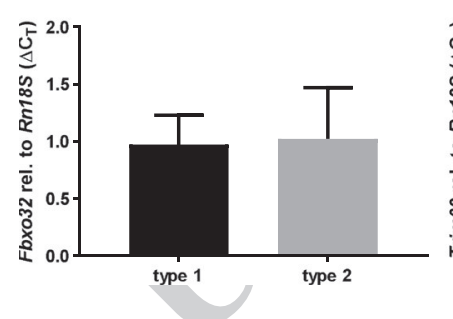

O
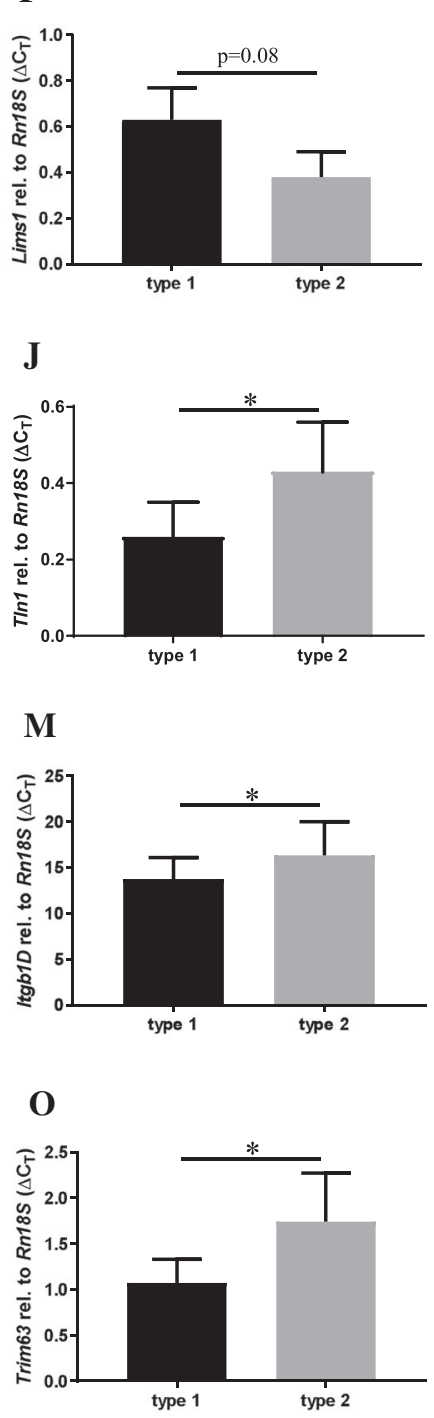

$\mathbf{J}$

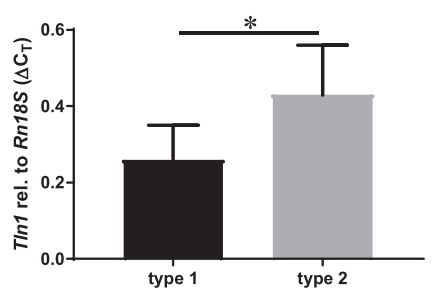

M

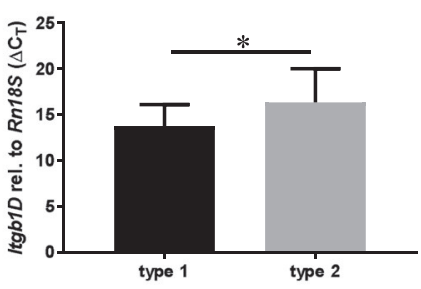

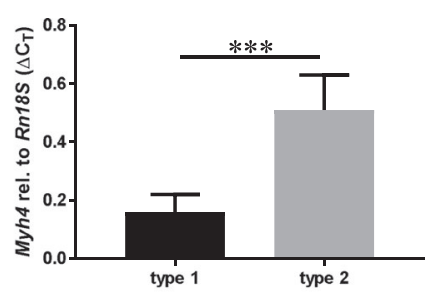

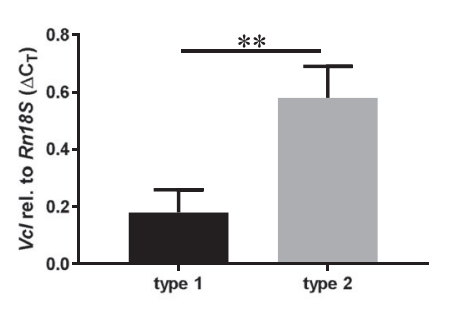

C

G

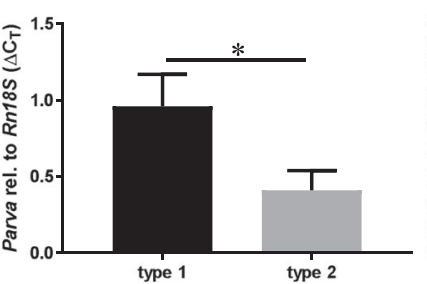

$\mathbf{H}$

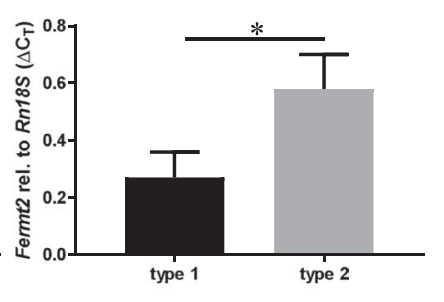

$\mathbf{K}$

\section{D}
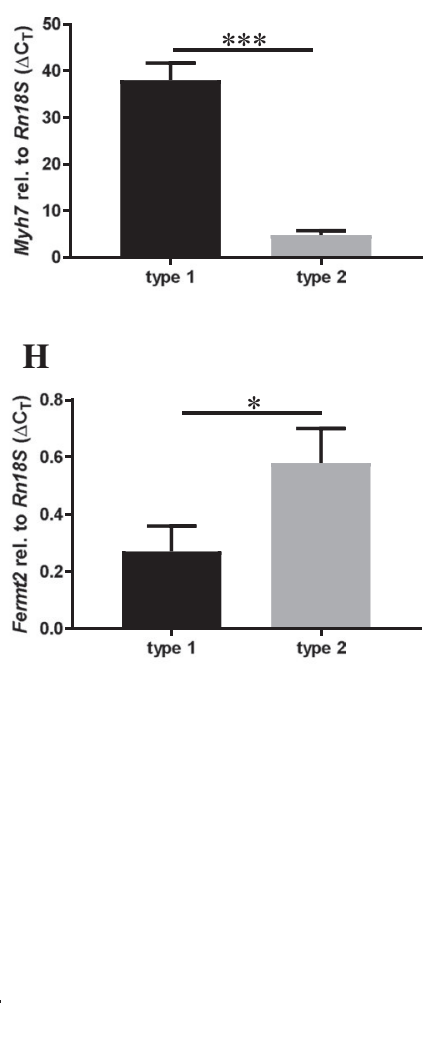

Fig. 6 Gene expression analysis in type 1 and type 2 fiber form lasermicrodissected control soleus muscles. a Myhl was significantly higher in type 2 compared to type 1 fibers. b $M y h 2$ was significantly higher in type 2 compared to type 1 fibers. c $M y h 4$ was significantly higher in type 2 compared to type 1 fibers. d $M y h 7$ was significantly higher in type 1 compared to type 2 fibers. e $I l k$ was significantly higher in type 2 compared to type 1 fibers. f Lims 1 tended to be higher in type 1 compared to type 2 fibers. g Parva was significantly higher in type 1 compared to type 2 fibers. h Fermt 2 was significantly higher in type 2 compared to type 1 fibers. i Xirp 2 was significantly higher in type 1 compared to type 2 fibers. j $T \ln 1$ was significantly higher in type 2 compared to type 1 fibers. $\mathbf{k ~}$ Vcl was significantly higher in type 2 compared to type 1 fibers. 1 Itga 7 was significantly higher in type 2 compared to type 1 fibers. m $\operatorname{Itg} b 1 D$ was significantly higher in type 2 compared to type 1 fibers. n Fbxo32 did not show any differences between type 1 and type 2 fibers. o Trim63 was significantly higher in type 2 compared to type 1 fibers. These data from control soleus muscle demonstrate that Cstms genes show clear fiber type-dependent expression patterns under basal conditions. $* p<0.05, * * p<0.01, * * * p<0.001$

\begin{tabular}{|l|l|l|l|l|}
\hline Journal : Large 18 & Article No : 3026 & Pages : 18 & MS Code : CMLS-D-18-01314 & Dispatch : 29-1-2019 \\
\hline
\end{tabular}


analysis and DNA gel electrophoresis. Additionally, we verified MT-PCR amplicons by sequencing (figure S5).

\section{Mechanical loading and unloading conditions alter Cstms gene expression in a fiber type-dependent manner}

With significant differences between fiber type-specific Cstms gene expression profiles under sedentary conditions, we next studied the influence of mechanical loading (WBV) vs. mechanical unloading (IML) on fiber type-specific Cstms expression, because mechanical cues trigger fiber type-specific adaptations via Cstms. We thus examined if the impact of altered mechanical signaling conditions influenced the expression of Cstms genes.

We first analyzed expression levels of four myosin heavychain-coding genes Myh1, Myh2, Myh4, and Myh7. Figure 7a demonstrates that WBV did not influence $M y h l$ gene profiles in type 1 and type 2 fibers. However, IML results in significantly increased $M y h l$ expression levels in type 1 fibers compared to control- and WBV-treated type 1 fibers. Type 1 fiber-specific IML-induced $M y h l$ expression was significantly higher compared to type 2 fibers. Interestingly, $M y h 1$ expression levels were unaltered in type 2 fibers. Myh2 expression did not show any differences between fiber types and interventions (Fig. 7b). WBV and IML both significantly increased Myh4 expressions in type 1 fibers compared to control conditions and compared to WBV and IML conditions in type 2 fibers (Fig. 7c). Myh7 gene expressions did not change in either fiber type regardless of loading condition (Fig. 7d).

Figure 7e underlines the mechanical responsiveness of the $I l k$ gene. $I l k$ was significantly induced by WBV in type 1 and 2 fibers compared to control and IML. Further, WBV may trigger higher Ilk expressions in type 1 compared to type 2 fibers. IML significantly reduced $I l k$ gene expressions in both fiber types compared to WBV, but not compared to sedentary control. The Lims 1 gene expression levels tended to increase in type 1 fibers upon, whereas IML induced significantly lower Lims 1 expression in type 1 fibers compared to control and WBV (Fig. 7f). Interestingly, WBV evoked a significantly higher Lims 1 expression in type 1 compared to type 2 fibers (Fig. 7f). In contrast, IML induced a significant reduction of Lims 1 expression in type 1 compared to type 2 fibers (Fig. 7f). Comparable to Ilk and Lims 1 , WBV significantly increased Fermt 2 expression in type 1 fibers and resulted in significantly higher levels compared to type 2 fibers (Fig. 7g). However, WBV resulted in significant reductions of Fermt 2 in type 2 fibers compared to type 1 fibers (Fig. 7g). Xirp2 tended to increase upon IML in type 1 fibers compared to control (Fig. 7h), whereas WBV did not have any influence. IML induced significantly higher Xirp2 expression in type 2 compared to type 1 fibers and significantly increased type 2-specific Xirp2 levels compared to WBV condition (Fig. 7h). WBV tended to be associated with increased Tln 1 expression only in type 1 fibers (Fig. 7i). $V c l$ was highly responsive to either intervention. Type 1 and type 2 fibers demonstrated significantly increased $\mathrm{Vcl}$ levels upon both WBV and IML compared to control (Fig. 7j). WBV was also associated with increased Itga7 expression in both type 1 and type 2 fibers compared to control and to IML (Fig. 7k). Itgb1D expression was similar for the different fiber types and not influenced by loading conditions (Fig. 71).

SkM disuse promotes protein breakdown-associated genes Fbxo32 and Trim 63 [4, 31]. We finally studied the influence of altered mechanical input on these genes in a fiber type-dependent manner. We found that WBV significantly reduced Fbxo 32 in type 1 fibers, whereas WBV induced Fbxo32 in type 2 fibers compared to control even more dramatically (Fig. $7 \mathrm{~m}$ ). IML significantly induced Fbxo 32 only in type 2 fibers and compared to type 2 fibers of control group (Fig. $7 \mathrm{~m}$ ). We only found a tendency for Trim63 to increase in type 1 fibers upon WBV compared to control (Fig. 7n). Finally, we studied SkM protein biosynthesis-related gene markers. We focused on mTor and $p 70 \mathrm{~s} 6 \mathrm{kbl}$ genes, because these have been shown to play major roles in protein biosynthesis initiation [32]. In line with Cstms genes, also $m$ Tor and $p 70 s 6 \mathrm{~kb} 1$ gene expressions were significantly increased upon WBV in both fiber types, with significantly higher levels in type 1 compared to type 2 fibers.

\section{Nuclear factor of activated T cell (NFATc1) overexpression in primary mouse skeletal muscle cells does not alter Ctsms gene expressions}

To explore the Cstms gene regulation mechanistically, we overexpressed NFATc1 in primary mouse myotubes to induce a switch towards slow MyHCs. Figure 8a-d demonstrates the successful overexpression of NFATc1 and a switch towards a slow MyHC program. The fast MyHCcoding Myhl and Myh4 gene expressions were significantly reduced, whereas the $M y h 2$ gene coding for the slow-like MyHC 2A [33] was significantly increased. Interestingly, we did not observe changes in central Cstms protein-coding genes (Fig. 8e). Hence, the induction of a slow MyHC program via the prominent NFATc1 pathway does not result in changes of Cstms gene expression profiles.

\section{Knockdown of integrin-linked kinase in differentiated muscle cells results in switch towards a slow-myosin heavy-chain program}

Because we did not observe changes in Cstms gene expressions upon slow MyHC program induction in differentiated muscle cells (C2C12 myotubes), we asked whether a

\begin{tabular}{|l|l|l|l|l|}
\hline Journal : Large 18 & Article No : 3026 & Pages : 18 & MS Code : CMLS-D-18-01314 & Dispatch : 29-1-2019 \\
\hline
\end{tabular}



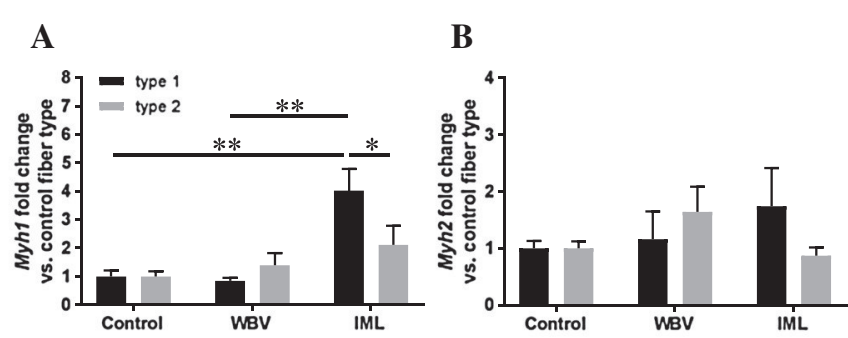

C

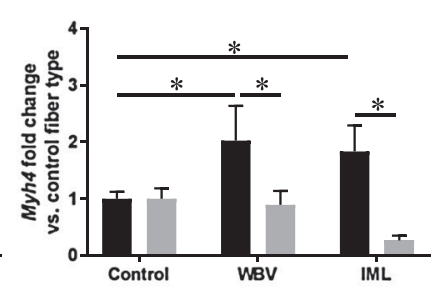

D

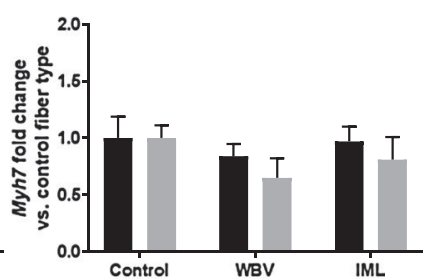

G

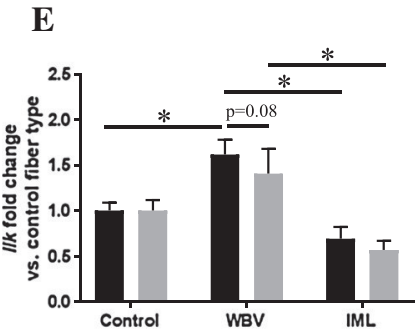

F
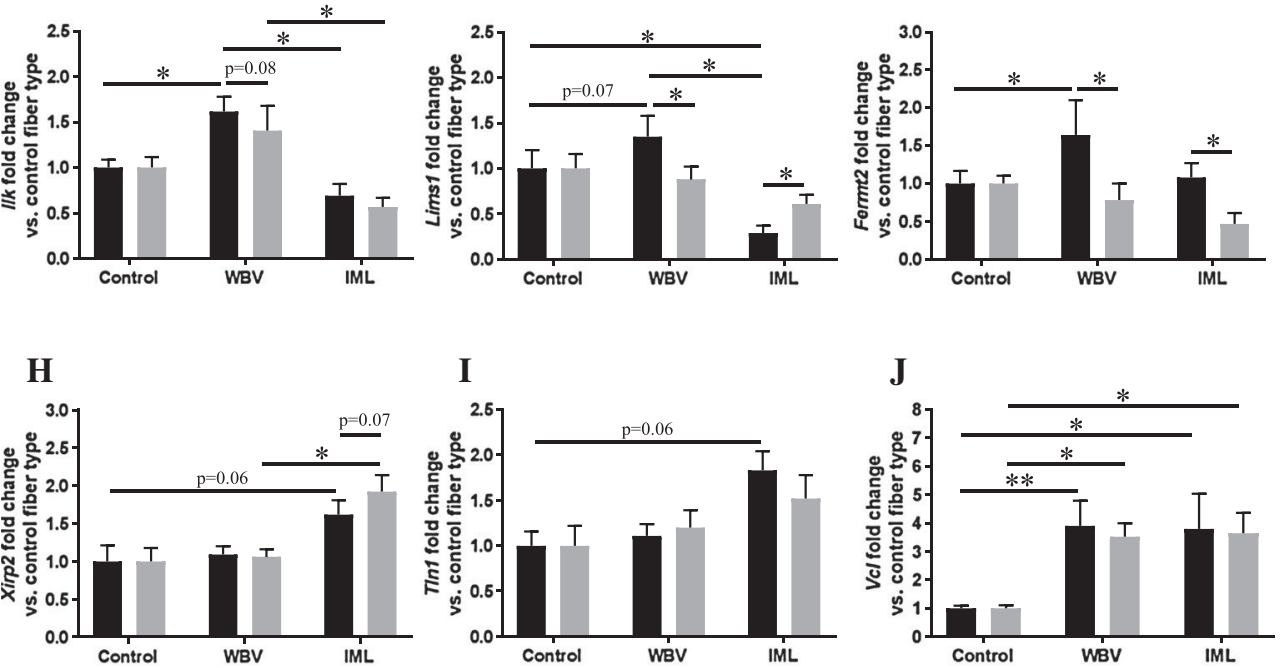

K

L
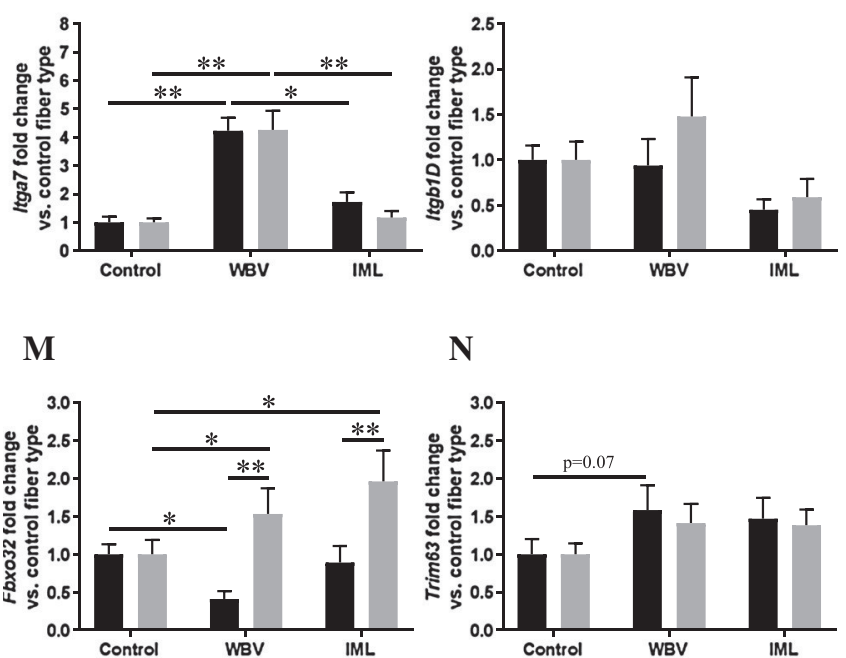

N

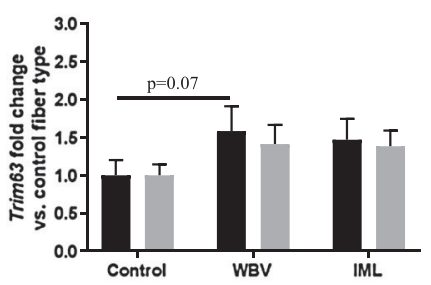

O

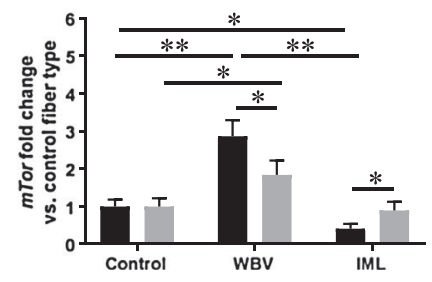

$\mathbf{P}$

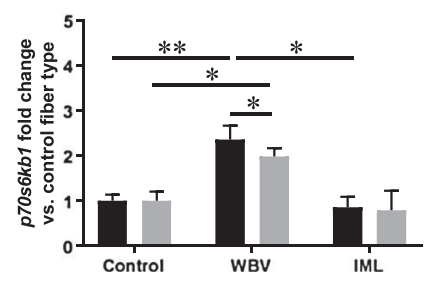


4Fig. 7 Gene expression analysis in type 1 and type 2 fiber form lasermicrodissected soleus muscles subjected to mechanical loading and unloading. Control type 1 and type 2 fiber gene expressions were set to ' 1 ' and WBV- and IML-related type 1 and type 2 gene expression data are given in relative changes to control values. a $M y h 1 ; \mathbf{b} M y h 2$; c Myh4; d Myh7; e Ilk; f Lims $1 ; \mathbf{g}$ Fermt2; h Xirp2; i Tln1; j Vcl; k Itga7; I Itgb1D; $\mathbf{m}$ Fbxo32; $\mathbf{n}$ Trim63; o mTor; $\mathbf{p}$ p70s6kb1. * $p<0.05$, $* * p<0.01$

knockdown of a central Cstms gene would initiate a change in MyHC program. To this end, we used an siRNA approach to knockdown Ilk in $\mathrm{C} 2 \mathrm{C} 12$ myotubes (Fig. 9a, b). The Ilk knockdown resulted in a reduction of protein biosynthesis-stimulating signaling molecules $\mathrm{p}-\mathrm{mTOR}^{\mathrm{Ser} 2448}$ and p-P70S6K ${ }^{\text {Thr389 }}$ relative to their unphosphorylated forms (Fig. 9b). Of note, we found that $M y h 7$ was significantly increased upon Ilk knockdown, whereas Myh1, Myh2, and Myh4 were significantly reduced (Fig. 9c). Furthermore, we observed reduced Fbxo32 and Trim63 levels upon Ilk knockdown. These data demonstrate that ILK is a critical determinant of a central fiber type characteristic, the MyHC expression level.

\section{Discussion}

SkM fibers are constantly exposed to mechanical forces. Consequently, the process of mechanotransduction is crucial for physiological and remodeling properties of SkM fibers and tissues. SkM fibers sense mechanical forces and respond to them to maintain their integrity, molecular architecture and signaling characteristics. Thereby, multi-facetted mechanotransduction enables SkM fibers to distinguish between smaller and larger mechanical impacts resulting in respective molecular adaptations. To exemplify this multi-facetted mechanotransduction, larger mechanical inputs by externally generated forces (e.g., resistance training or whole-body vibration, larger impacts) activate SkM hypertrophy signals, while mechanical cutback conditions elicit smaller mechanical impacts (e.g., immobilization, disuse) triggering SkM atrophy signals.

Cstms are specialized structures for mechanosensing and mechanical signal amplification of striated muscle fibers [34]. Many Cstms components are involved in the control of physiological SkM development and fiber integrity [6]. In particular, ILK [8, 35], ITGA7 [10], ITGB1D [36, 37], FERMT2 [13] or TLN [11] is indispensable for SkM physiology and integrity. Cstms connect the outermost myofibrils to the sarcolemma at each $\mathrm{Z}$ disc and enable myofibrils to transmit contraction forces throughout the entire fiber [38, 39]. SkM tissue is composed of fibers portraying a broad spectrum of morphological, biophysical and metabolic properties [1]. Interestingly, the expression patterns of central mechanosensors and -amplifiers of the Cstms network are completely unexplored in a fiber type population-specific manner. However, decoding Cstms networks in a fiber typedependent manner will allow for a more complex and precise understanding of physiological control of SkM adaptations to any stimuli.

We developed a multiplex tandem PCR approach coupled to laser microdissection-assisted enrichment of SkM fiber types to study Cstms protein and gene regulation in detail under basal sedentary as well as under conditions of increased (WBV) vs. reduced (IML) mechanical impacts.

Using imaging techniques, we found that PINCH-1, TLN and beta-XIN predominantly localized in type 1 fibers, while ILK, VCL, and ITGA7 were predominantly localized in type 2 fibers demonstrating clearly that defined Cstms components are expressed in a fiber type-dependent manner. PARVB and ITGB1D do not show obvious distributions. This provided first evidence that different SkM fiber types manage mechanotransduction differently. We assume that these fiber type-dependent expression levels are important to manage high contraction force-induced physical forces in type 2 fibers and to convert them into downstream signals that allow specific adaptations instead of damage. In low contraction force-developing type 1 fibers, Cstms are nevertheless sufficiently composed to initiate and to control downstream signals, whereas the magnitude of signal conversion seems to be lower compared to type 2 fibers. We next used an LMD approach to collect highly enriched type 1 vs. type 2 fibers from mouse soleus muscles. We focused on ILK and TLN because these proteins are highly enriched in SkM. We demonstrate that TLN accumulates in type 1 fibers, whereas we detected ILK predominantly in type 2 fibers, which is consistent with our histological analyses. As shown previously [40] and confirmed by our results, we used $\alpha$-actinin- 3 as an internal control. These protein-based data demonstrate clearly that type 1 and type 2 fibers possess functionally distinct adhesion complexes to manage mechanotransduction and hence downstream localized signaling cues, consequently adapting differentially to stimulations.

To verify our protein and localization data, we analyzed Cstms gene fiber type specifically. Because SkM fibers are low in mRNA, we pre-amplified cDNA instead of RNA and developed a highly sensitive MT-PCR approach to study a large number of GOIs from a single isolation of defined fiber populations corresponding to $3 \times 10^{6} \mu \mathrm{m}^{2}$. We examined the linearity of our pre-amplification approach to exclude any bias in downstream gene analysis and found that our pre-amplifications were linear with neither absolute nor $\log _{10}$-transformed $\Delta \Delta C_{\mathrm{T}}$ values exceeding a defined threshold of \pm 1.5 or \pm 0.452 , respectively [30] nor did we observe primer dimer formation in DNA gel electrophoresis. We started with fiber type-specific LMD-MT-qPCR analyses of sedentary control SkM and identified $M y h 7$ as a type 1 fiber-specific gene and Myhl,Myh2 and Myh4 (all

\begin{tabular}{|l|l|l|l|l|}
\hline Journal : Large 18 & Article No : 3026 & Pages : 18 & MS Code : CMLS-D-18-01314 & Dispatch : 29-1-2019 \\
\hline
\end{tabular}



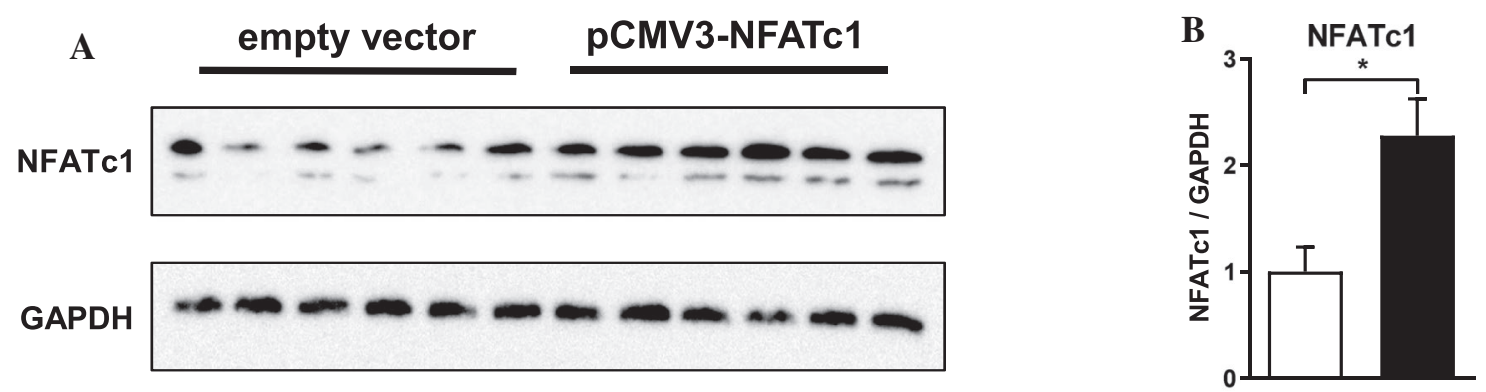

C

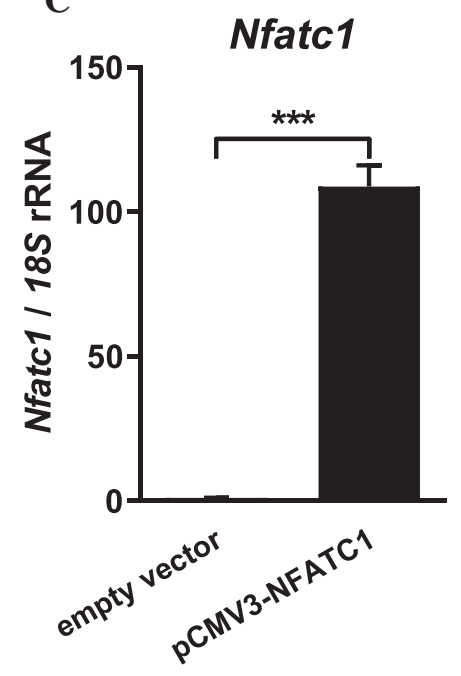

D

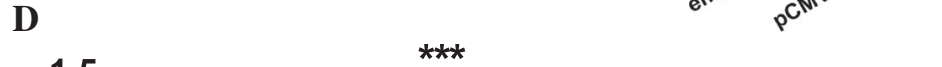

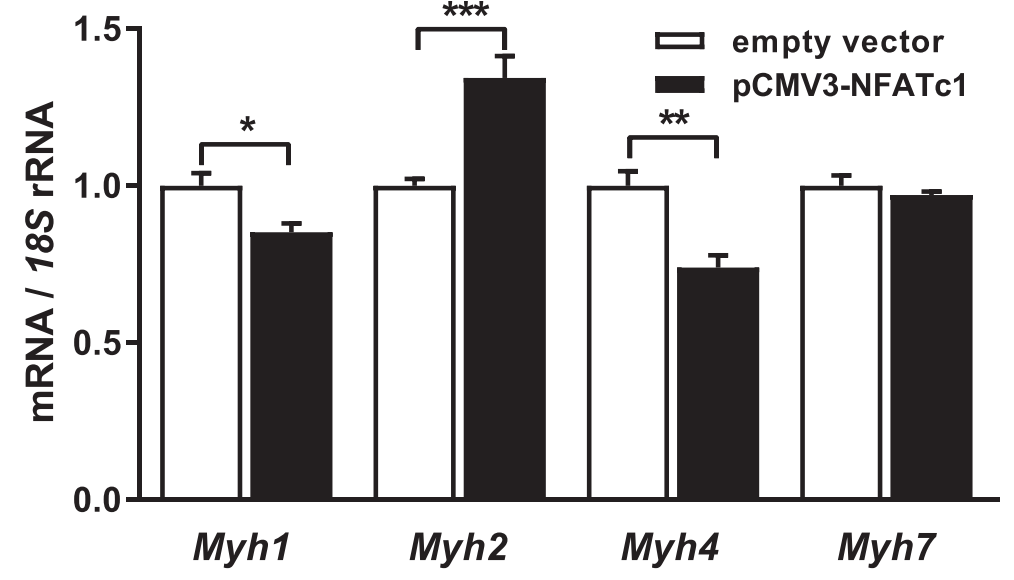

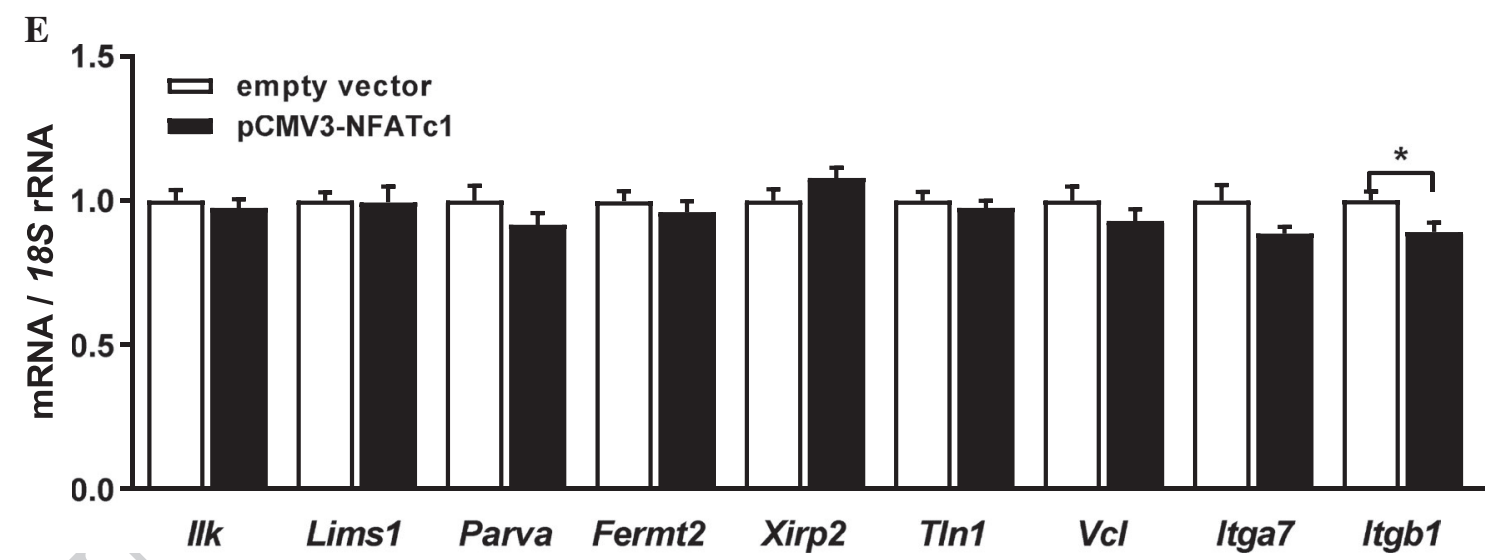

Fig. 8 NFATc1 overexpression in mouse primary skeletal muscle cells does not change Cstms gene expressions. a Western blot analysis shows successful overexpression of NFATc1 by means of the pCMV3-NFATc1 vector. GAPDH served as loading control. b Quantifications of NFATc1 protein levels. c Nfatcl gene expression quan- type 2 fibers) as type 2 fiber-specific markers, confirming the success of our fiber population sampling. We found that Ilk, Fermt2, Tln1, Vcl, Itga7, and Itgb1D were expressed at significantly higher levels in type 2 compared to type 1 fibers, whereas Lims 1, Parva, and Xirp2 showed significantly higher expressions in type 1 compared to type 2 fibers. tification. d $M y h 1, M y h 2, M y h 4$, and $M y h 7$ gene expressions demonstrate a switch towards slow-myosin heavy-chain gene programs. e Cstms gene analysis in NFATc1-overexpressing compared to control transfected cells. $* p<0.05, * * p<0.01, * * * p<0.001$

These data were consistent with our localization studies, except for $T \ln 1$, because we localized TLN primarily in type 1 fibers. One reason for this discrepancy is that the used antibody recognized both TLN1 and TLN2 isoforms, which might explain the observed differences. Because such analyses have never been carried out before, we cannot 

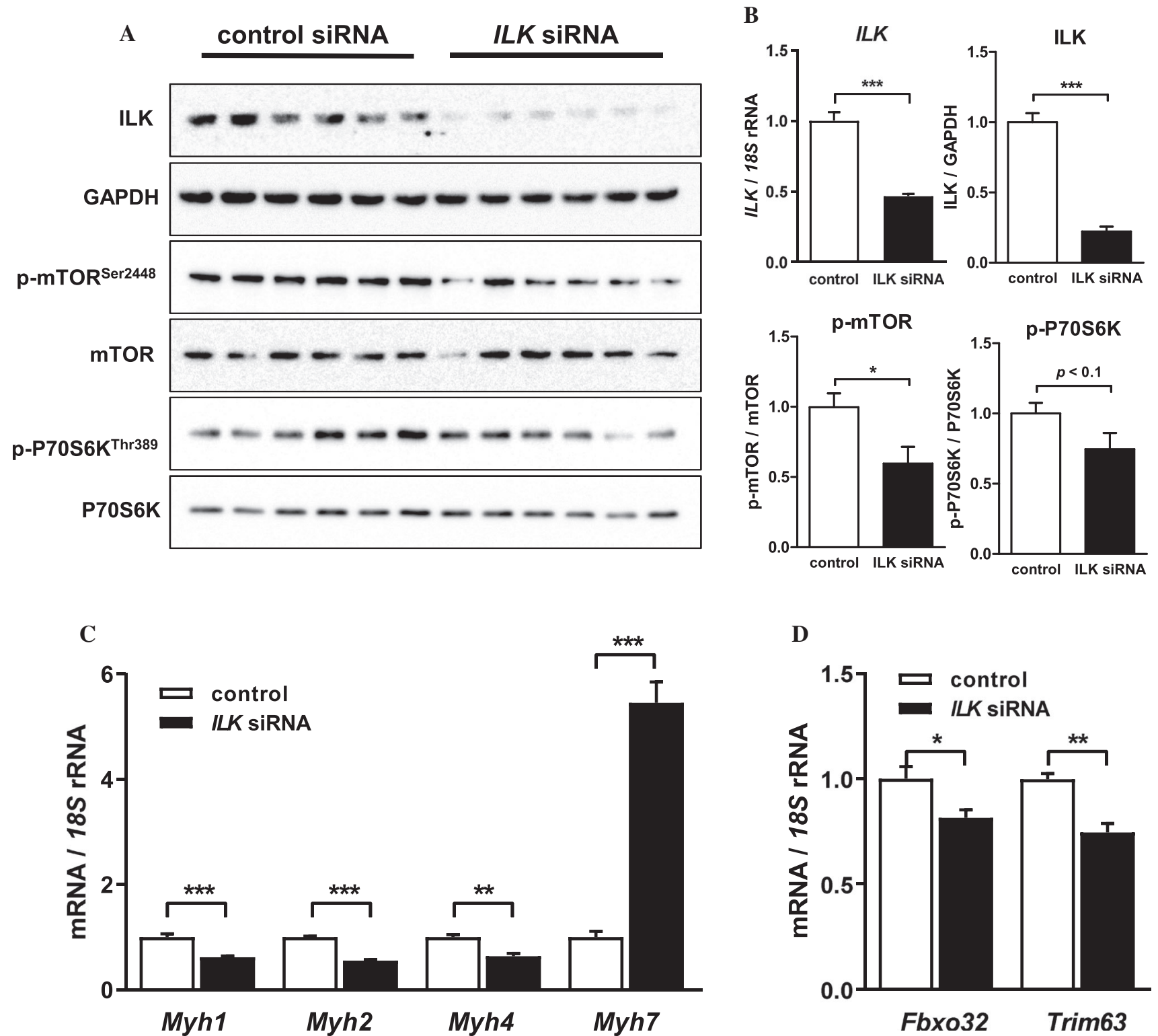

Fig. 9 Knockdown of integrin-linked kinase in $\mathrm{C} 2 \mathrm{C} 12$ myotubes results in switch of myosin heavy chain program. a Western blot analysis of ILK and protein biosynthesis-related signaling molecules (mTOR and P70S6K) in Ilk-deficient and control cells. GAPDH served as loading control. b $I l k$ and ILK, p-mTOR ${ }^{\text {Ser2448, }}$ compare these data to the literature. However, they provide a basis as to why SkMs with a high proportion of type 2 fibers (e.g., EDL or gastrocnemius) are more prone to muscle dystrophy-like phenotypes when defined Cstms genes, such Ilk [8] or Itga7 [10], are deleted or mutated. This association adds to our understanding how single SkM fibers build their Cstms adhesion structures to control downstream localized signaling cues, e.g., fiber growth-promoting (e.g., Akt/ PKB [7, 41]) and -surviving (e.g., Bcl-2 [7]) signals. We did not find fiber type-specific differences of Fbxo32 gene and p-P70S6K ${ }^{\text {Thr389 }}$ quantifications. c Myhl, Myh2, Myh4, and Myh7 gene expressions in Ilk-deficient and control cells demonstrate an Ilkdependent switch towards slow-myosin heavy-chain expressions. d Muscle atrophy marker genes Fbxo32 and Trim63 are reduced upon Ilk knockdown

expression, whereas Trim63 expression was significantly higher expressed in type 2 compared to type 1 fibers, which is in line with previous studies [42], indicating that fiber types regulate protein turnover differentially.

Our next analyses determined whether type 1 and type 2 fibers modulate their Cstms gene expression differently in response to loading and unloading. We used a mouse model of WBV and IML and found that the gastrocnemius CSA increased by $16 \%$ upon WBV and decreased by $21 \%$ upon IML. We demonstrate that $M y h 1$ and $M y h 4$ are specifically

\begin{tabular}{|l|l|l|l|l|}
\hline Journal : Large 18 & Article No : 3026 & Pages : 18 & MS Code : CMLS-D-18-01314 & Dispatch : 29-1-2019 \\
\hline
\end{tabular}


upregulated upon IML in type 1 fibers, while type 2 fibers remain unaffected. Myh2 and $M y h 7$ were stable in each fiber type regardless of intervention. Calcium $\left(\mathrm{Ca}^{2+}\right)$ signaling involved in fiber type maintenance and shifting [43] seemed to alter under IML conditions favoring a shift towards fast fiber type ratios even in slow fibers, indicating that a 'pure' fiber with respect to $M y h$ gene expressions does not exist. $I l k$ was significantly increased upon WBV in type 1 fibers compared to sedentary control and IML conditions and showed higher type 1 fiber levels upon WBV compared to type 2 fibers. Recently, Ilk was shown to be upregulated in SkM tissue upon mechanical stimulation, which fits with our analyses [44]. Our data reveal that also Lims1, Fermt2, $\mathrm{Vcl}$, and Itga7 expressions were increased predominantly in type 1 fibers upon WBV intervention. Furthermore, we found reduced Lims 1, Fermt2, and Itga7 expressions upon IML with defined but heterogeneous fiber type specificities. Interestingly, $\mathrm{Vcl}$ was also significantly increased upon IML in a fiber type-independent manner, but $T \ln 1 /$ ItgblD expressions remained unaffected.

These data provide evidence that SkM Cstms assembly depends on externally applied stimuli and that the composition of Cstms can change rapidly in a fiber type-dependent manner upon defined stimuli to manage downstream adaptation processes. Most of the genes increased upon WBV, whereas IML downregulated their expression levels. Important to note is that type 1 fibers showed a more frequent adjustability of specific Cstms adaptations than type 2 fibers uncovering that type 1 fibers are more sensitive to changes of their external mechanical stimuli compared to type 2 fibers. Consequently, our findings introduce the novel concept that type 1 and type 2 fibers generate distinct but defined Cstmsdependent response matrices [45] to manage increased or decreased mechanical input. This could serve as a backbone to explain, why defined Cstms deletions result in SkM-specific dysfunction [10] and why distinct SkM fiber types show divergent metabolic adaptations [2]. These two observations are likely to be controlled by Cstms-dependent signaling pathways involved in SkM fiber integrity and regulation of metabolic adaptations $[7,8,40]$.

We further studied genes coding for protein breakdownassociated [4] Fbxo32 and Trim63 genes as well as for protein biosynthesis-associated $m T o r$ and $p 70 s 6 \mathrm{kbl}$ genes [32] to study the influence of the applied interventions on these SkM adaptation pathways. Fbxo32 was increased in type 2 compared to type 1 fibers upon both WBV and IML, whereas the same condition resulted in significantly reduced Fbxo32 levels in type 2 fibers compared to control condition. This is in contrast to a recent study demonstrating that disuse increases $F b x o 32$ in type 1, but reduces it in type 2 fibers [27]. Interestingly, we did not observe any significant changes in Trim63. mTor gene expression was significantly increased upon WBV in both fiber types compared to control condition, whereas $m$ Tor showed higher expressions in type 1 compared to type 2 fibers. IML reduced $m$ Tor expressions in type 1 fibers compared to both control and WBV conditions. We found similar results for $p 70 \mathrm{~s} 6 \mathrm{kbl}$ gene expressions, which were significantly increased in both fiber types upon WBV compared to control condition. These data reveal that critical protein breakdown and biosynthesis-associated genes respond differentially to mechanical loading and unloading and support fiber type-specific protein synthesis [2], metabolism [46], and mass-regulation [1].

Our final analyses dealt with the questions whether an induction of the slow MhHC program changes Cstms expressions and vice versa whether manipulated Cstms expressions guide the MyHC program. Therefore, we first overexpressed NFATc1 in vitro to induce the slow MyHC program and analyzed Cstms genes. It was demonstrated that the calcineurin-NFAT pathway has a major role as activator and repressor of different $M y h$ genes in SkM. SkM in vivo transfection with NFATc1 increased MyHC 1 and decreases MyHC 2B promoter activity [47]. Accordingly, the NFAT inhibitor VIVIT increased the MyHC 2X and 2B-coding genes Myhl and Myh4 in adult SkM [47]. Since we could not induce transitions of MyHC-coding genes in $\mathrm{C} 2 \mathrm{C} 12$ cells, we used mouse primary skeletal muscle cells for NFATc1 overexpression. NFATc1 transfected primary myotubes showed the expected clear downregulation of Myhl and $M y h 4$, while $M y h 2$, which is expressed in fibers with a high oxidative enzyme profile similar to that of $M y h 7$ expressing fibers [33], showed a significant upregulation. Upon successful activation of the slow gene program, we analyzed the transcriptional Cstms expression profiles. However, we could not detect any change of Cstms transcriptional activity.

We, therefore, asked whether the Cstms profile of the SkM fiber determines the MyHC profile. We decided to knockdown $I l k$, because it represents the major regulator of integrin-mediated signaling [7], is crucial for SkM integrity [8], and is responsible for activation of downstream Akt signaling in SkM [8]. Upon successful downregulation of Ilk gene and protein levels, we observed decreased

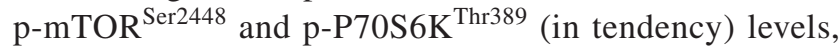
which are responsible for SkM fiber hypertrophy and cell growth [32]. We could further show that Ilk as a central regulator of the IPP complex affects MyHC gene expression. The fast MyHC-coding genes (Myh1, Myh2, Myh4) were significantly downregulated, whereas $M y h 7$, which is predominantly present in slow fibers, was more than fivefold upregulated. Accordingly, this result is in line with the outlined higher Ilk gene and protein levels in type-II fibers.

In conclusion, our study provides detailed insights into the formation of the specialized fiber type-dependent Cstms adhesion clusters. We demonstrate that different mechanosensors and -amplifiers of the IRC/IPP Cstms show specific type 1 or type 2 fiber expressions under sedentary 
control and (un)loading conditions. These data highlight the existence of defined but distinct mechano-sensitive clusters in type 1 and type 2 fibers, which can be remodeled by external impacts. We further conclude that Cstms rather determine the composition of the sarcomeric cytoskeleton than vice versa. We, therefore, suggest that Cstms are involved in SkM fiber type transitions following (un)loading conditions. Our study systematizes the complexity between type 1 and type 2 fibers in terms of controlling and managing mechanotransduction and potentially downstream localized/triggered signaling pathways. Finally, our data break ground for the detailed analysis of SkM tissue adaptations to mechanical loading and unloading conditions on a fiber type population-specific basis. Our approaches will shed light on the regulation of SkM integrity and physiology regulation in healthy as well as diseased tissues. Further, our data demonstrate that fiber types adapt differentially to external stimuli highlighting the necessity for careful discriminations between fiber types.

Acknowledgements This study was supported by the German Sport University (920122 and 920119), the Federal Institute of Sports Sciences (IIA1-070114/13), and a KU Leuven Grant (ZKD2412) to F.S. The authors thank Bianca Collins and Mojgan Ghilav (both Department of Molecular Cellular Sport Medicine, German Sport University Cologne) for their excellent technical assistance. Professor Rik Lories (Skeletal Biology and Engineering Research Center, KU Leuven) is highly acknowledged for critically reading the manuscript.

Author contributions FS designed the study. SM, MV and FS carried out experiments, performed statistics and interpreted the data. WB contributed to data interpretation and reagents. SM and FS prepared the figures. FS wrote the manuscript. All authors approved the final version of the manuscript.

\section{Compliance with ethical standards}

Conflict of interest The authors declare that they have no competing interests.

\section{References}

1. Schiaffino S, Reggiani C (2011) Fiber types in mammalian skeletal muscles. Physiol Rev 91:1447-1531. https://doi.org/10.1152/ physrev.00031.2010

2. Goodman CA, Kotecki JA, Jacobs BL, Hornberger TA (2012) Muscle fiber type-dependent differences in the regulation of protein synthesis. PLoS One 7:e37890. https://doi.org/10.1371/journ al.pone. 0037890

3. Tidball JG (2005) Mechanical signal transduction in skeletal muscle growth and adaptation. J Appl Physiol 98:1900-1908. https:// doi.org/10.1152/japplphysiol.01178.2004

4. Sandri M (2008) Signaling in muscle atrophy and hypertrophy. Physiology (Bethesda) 23:160-170. https://doi.org/10.1152/physi ol.00041.2007

5. Burkholder TJ (2007) Mechanotransduction in skeletal muscle. Front Biosci 12:174-191
6. Pingel J, Suhr F (2017) Are mechanically sensitive regulators involved in the function and (patho)physiology of cerebral palsy-related contractures? J Muscle Res Cell Motil 38:317330. https://doi.org/10.1007/s10974-017-9489-1

7. Legate KR, Montañez E, Kudlacek O, Fässler R (2006) ILK, PINCH and parvin: the tIPP of integrin signalling. Nat Rev Mol Cell Biol 7:20-31. https://doi.org/10.1038/nrm1789

8. Wang H-V, Chang L-W, Brixius K et al (2008) Integrin-linked kinase stabilizes myotendinous junctions and protects muscle from stress-induced damage. J Cell Biol 180:1037-1049. https ://doi.org/10.1083/jcb.200707175

9. Taverna D, Disatnik MH, Rayburn H et al (1998) Dystrophic muscle in mice chimeric for expression of alpha5 integrin. $\mathrm{J}$ Cell Biol 143:849-859

10. Mayer U, Saher G, Fässler R et al (1997) Absence of integrin alpha 7 causes a novel form of muscular dystrophy. Nat Genet 17:318-323. https://doi.org/10.1038/ng1197-318

11. Conti FJ, Monkley SJ, Wood MR et al (2009) Talin 1 and 2 are required for myoblast fusion, sarcomere assembly and the maintenance of myotendinous junctions. Development 136:35973606. https://doi.org/10.1242/dev.035857

12. Shear CR, Bloch RJ (1985) Vinculin in subsarcolemmal densities in chicken skeletal muscle: localization and relationship to intracellular and extracellular structures. J Cell Biol 101:240-256

13. Dowling JJ, Vreede AP, Kim S et al (2008) Kindlin-2 is required for myocyte elongation and is essential for myogenesis. BMC Cell Biol 9:36. https://doi.org/10.1186/1471-2121-9-36

14. Gehlert S, Suhr F, Gutsche K et al (2014) High force development augments skeletal muscle signalling in resistance exercise modes equalized for time under tension. Pflugers Arch 467:1343-1356. https://doi.org/10.1007/s00424-014-1579-y

15. Bottinelli R, Canepari M, Pellegrino MA, Reggiani C (1996) Force-velocity properties of human skeletal muscle fibres: myosin heavy chain isoform and temperature dependence. J Physiol 495(Pt 2):573-586

16. Bottinelli R, Schiaffino S, Reggiani C (1991) Force-velocity relations and myosin heavy chain isoform compositions of skinned fibres from rat skeletal muscle. J Physiol 437:655-672

17. Gilliver SF, Degens H, Rittweger J et al (2009) Variation in the determinants of power of chemically skinned human muscle fibres. Exp Physiol 94:1070-1078. https://doi.org/10.1113/ expphysiol.2009.048314

18. Fluck M (2006) Functional, structural and molecular plasticity of mammalian skeletal muscle in response to exercise stimuli. J Exp Biol 209:2239-2248. https://doi.org/10.1242/jeb.02149

19. Suhr F, Brixius K, de Marées M et al (2007) Effects of shortterm vibration and hypoxia during high-intensity cycling exercise on circulating levels of angiogenic regulators in humans. $\mathrm{J}$ Appl Physiol 103:474-483. https://doi.org/10.1152/japplphysi ol.01160.2006

20. Mester J, Kleinöder H, Yue Z (2006) Vibration training: benefits and risks. J Biomech 39:1056-1065. https://doi.org/10.1016/j. jbiomech.2005.02.015

21. Lin C-I, Huang W-C, Chen W-C et al (2015) Effect of wholebody vibration training on body composition, exercise performance and biochemical responses in middle-aged mice. Metabolism 64:1146-1156. https://doi.org/10.1016/j.metab ol.2015.05.007

22. Huang C-C, Tseng T-L, Huang W-C et al (2014) Whole-body vibration training effect on physical performance and obesity in mice. Int J Med Sci 11:1218-1227. https://doi.org/10.7150/ ijms. 9975

23. Warner SE, Sanford DA, Becker BA et al (2006) Botox induced muscle paralysis rapidly degrades bone. Bone 38:257-264. https ://doi.org/10.1016/j.bone.2005.08.009

\begin{tabular}{|l|l|l|l|l|}
\hline Journal : Large 18 & Article No: 3026 & Pages : 18 & MS Code : CMLS-D-18-01314 & Dispatch : 29-1-2019 \\
\hline
\end{tabular}


24. Eng CM, Smallwood LH, Rainiero MP et al (2008) Scaling of muscle architecture and fiber types in the rat hindlimb. J Exp Biol 211:2336-2345. https://doi.org/10.1242/jeb.017640

25. Hawke TJ, Atkinson DJ, Kanatous SB et al (2007) Xin, an actin binding protein, is expressed within muscle satellite cells and newly regenerated skeletal muscle fibers. Am J Physiol Cell Physiol 293:C1636-C1644. https://doi.org/10.1152/ajpcell.00124.2007

26. Choi S, Gustafson-Wagner EA, Wang Q et al (2007) The intercalated disk protein, mXinalpha, is capable of interacting with beta-catenin and bundling actin filaments [corrected]. J Biol Chem 282:36024-36036. https://doi.org/10.1074/jbc.M707639200

27. Vanderburg CR, Clarke MSF (2013) Laser capture microdissection of metachromatically stained skeletal muscle allows quantification of fiber type specific gene expression. Mol Cell Biochem 375:159-170. https://doi.org/10.1007/s11010-012-1538-x

28. Ichinoseki-Sekine N, Yoshihara T, Kakigi R et al (2012) Fibertype specific expression of $\alpha$-actinin isoforms in rat skeletal muscle. Biochem Biophys Res Commun 419:401-404. https://doi. org/10.1016/j.bbrc.2012.02.034

29. Fleige S, Pfaffl MW (2006) RNA integrity and the effect on the real-time qRT-PCR performance. Mol Aspects Med 27:126-139. https://doi.org/10.1016/j.mam.2005.12.003

30. Noutsias M, Rohde M, Block A et al (2008) Preamplification techniques for real-time RT-PCR analyses of endomyocardial biopsies. BMC Mol Biol 9:3. https://doi.org/10.1186/1471-2199-9-3

31. Bodine SC, Baehr LM (2014) Skeletal muscle atrophy and the E3 ubiquitin ligases MuRF1 and MAFbx/atrogin-1. Am J Physiol Metab 307:E469-E484. https://doi.org/10.1152/ajpendo.00204 .2014

32. Bodine SC, Stitt TN, Gonzalez M et al (2001) Akt/mTOR pathway is a crucial regulator of skeletal muscle hypertrophy and can prevent muscle atrophy in vivo. Nat Cell Biol 3:1014-1019. https ://doi.org/10.1038/ncb1101-1014

33. Allen DL, Leinwand LA (2002) Intracellular calcium and myosin isoform transitions. Calcineurin and calcium-calmodulin kinase pathways regulate preferential activation of the IIa myosin heavy chain promoter. J Biol Chem 277:45323-45330. https://doi. org/10.1074/jbc.M208302200

34. Samarel AM (2005) Costameres, focal adhesions, and cardiomyocyte mechanotransduction. Am J Physiol Heart Circ Physiol 289:H2291-H2301. https://doi.org/10.1152/ajpheart.00749.2005

35. Gheyara AL, Vallejo-Illarramendi A, Zang K et al (2007) Deletion of integrin-linked kinase from skeletal muscles of mice resembles muscular dystrophy due to alpha 7 beta 1-integrin deficiency. Am J Pathol 171:1966-1977. https://doi.org/10.2353/ ajpath.2007.070555

36. van der Flier A, Kuikman I, Baudoin C et al (1995) A novel beta 1 integrin isoform produced by alternative splicing: unique expression in cardiac and skeletal muscle. FEBS Lett 369:340-344

37. van der Flier A, Gaspar AC, Thorsteinsdóttir S et al (1997) Spatial and temporal expression of the beta1D integrin during mouse development. Dev Dyn 210:472-486. https://doi. org/10.1002/(SICI)1097-0177(199712)210:4\%3c472:AID-AJA10 $\% 3$ e3.0.CO;2-9

38. Ervasti JM (2003) Costameres: the Achilles' heel of Herculean muscle. J Biol Chem 278:13591-13594. https://doi.org/10.1074/ jbc.R200021200
39. Estrella NL, Naya FJ (2014) Transcriptional networks regulating the costamere, sarcomere, and other cytoskeletal structures in striated muscle. Cell Mol Life Sci 71:1641-1656. https://doi. org/10.1007/s00018-013-1512-0

40. MacArthur DG, Seto JT, Raftery JM et al (2007) Loss of ACTN3 gene function alters mouse muscle metabolism and shows evidence of positive selection in humans. Nat Genet 39:1261-1265. https://doi.org/10.1038/ng2122

41. Kimura M, Murakami T, Kizaka-Kondoh S et al (2010) Functional molecular imaging of ILK-mediated Akt/PKB signaling cascades and the associated role of beta-parvin. J Cell Sci 123:747-755. https://doi.org/10.1242/jcs.052498

42. Moriscot AS, Baptista IL, Bogomolovas J et al (2010) MuRF1 is a muscle fiber-type II associated factor and together with MuRF2 regulates type-II fiber trophicity and maintenance. J Struct Biol 170:344-353. https://doi.org/10.1016/j.jsb.2010.02.001

43. Gehlert S, Bloch W, Suhr F (2015) $\mathrm{Ca}^{2+}$-dependent regulations and signaling in skeletal muscle: from electro-mechanical coupling to adaptation. Int J Mol Sci 16:1066-1095. https://doi. org/10.3390/ijms16011066

44. Chaillou T, Lee JD, England JH et al (2013) Time course of gene expression during mouse skeletal muscle hypertrophy. J Appl Physiol 115:1065-1074. https://doi.org/10.1152/japplphysi ol.00611.2013

45. Toigo M, Boutellier U (2006) New fundamental resistance exercise determinants of molecular and cellular muscle adaptations. Eur J Appl Physiol 97:643-663. https://doi.org/10.1007/s0042 1-006-0238-1

46. Greenhaff PL, Söderlund K, Ren JM, Hultman E (1993) Energy metabolism in single human muscle fibres during intermittent contraction with occluded circulation. J Physiol 460:443-453

47. McCullagh KJA, Calabria E, Pallafacchina G et al (2004) NFAT is a nerve activity sensor in skeletal muscle and controls activity-dependent myosin switching. Proc Natl Acad Sci USA 101:10590-10595. https://doi.org/10.1073/pnas.0308035101

48. Suhr F (2015) Detection of fusion events in mammalian skel-AQ5 etal muscle. Methods Mol Biol 1313:115-129. https://doi. org/10.1007/978-1-4939-2703-6_8

49. Greiwe L, Vinck M, Suhr F (2016) The muscle contraction mode determines lymphangiogenesis differentially in rat skeletal and cardiac muscles by modifying local lymphatic extracellular matrix microenvironments. Acta Physiol 217:61-79. https://doi. org/10.1111/apha.12633

50. Gehlert S, Suhr F, Gutsche K et al (2015) High force development augments skeletal muscle signalling in resistance exercise modes equalized for time under tension. Pflugers Arch 467:1343-1356. https://doi.org/10.1007/s00424-014-1579-y

51. Livak KJ, Schmittgen TD (2001) Analysis of relative gene expression data using real-time quantitative PCR and the 2(-Delta Delta C(T)) Method. Methods 25:402-408. https://doi.org/10.1006/ meth.2001.1262

Publisher's Note Springer Nature remains neutral with regard to jurisdictional claims in published maps and institutional affiliations. 